\title{
Microbiological and Physico-Chemical Parameters of the Danube River (Romanian- Bulgarian Sector) with Ichthyofaunal Diversity
}

\author{
${ }^{1}$ Mădălina-Andreea Ivan, ${ }^{1}$ Carmen Curuțiu, ${ }^{2}$ Nicolae Crăciun, ${ }^{3}$ Valentin \\ Jujea and ${ }^{4}$ Cristian-Emilian Pop
}

${ }^{1}$ Department of Microbiology, Faculty of Biology, University of Bucharest, 1-3 Aleea Portocalelor Str., 60101 Bucharest, Romania; ${ }^{2}$ Zoology Section, Department of Biochemistry and Molecular Biology, Faculty of Biology, University of Bucharest, 9195 Splaiul Independenței Str., 050095, Bucharest, Romania; ${ }^{3}$ Department of Geographic Information Systems, Faculty of Geography, University of Bucharest, 1 Nicolae Bălcescu Str., 010041, Bucharest, Romania; ${ }^{4}$ Department of Organic Chemistry, Biochemistry and Catalysis, Faculty of Chemistry, University of Bucharest, 90 Panduri Str., 050663, Bucharest, Romania. Corresponding authors: M.-A. Ivan m.ivan20@s.bio.unibuc.ro; C.-E. Pop cristian.pop@s.unibuc.ro

\begin{abstract}
Water and water habitats are essential to life and to the wellness of the communities that rely on them, as the Danube river is subject to human impact and vice versa, periodic surveys are required to determine the status of its pollution levels. In this work we used known microbiological indicators as well as physico-chemical ones to assess the ecotoxicological status of the Danube river from eleven locations partially forming the "Romanian-Bulgarian Danube Sector". Most of the microbial communities found, and their densities, could be explained by point and diffuse sources scattered along the sector such as discharged household and farm wastewater, as well as nearby agricultural areas where fertilizers have been used and then leaked in the water stream during rainfalls. However, microbial data on non-point sources that lead to diffuse pollution of surface waters could be linked to such parameters and furthermore shown a slight correlation with the current status of fish communities.
\end{abstract}

Key Words: water microbiology; ecotoxicology; Danube; wastewater; ichthyofauna; pollution indicators

\section{Introduction}

The Danube river has been subject to many pollution sources over the years, studies revealing sediments $(1,2)$ being able to highlight historical modifications of the major impacts over it, including agriculture fertilizers, pesticides and insecticides, as well as discharge of wastewater and other anthropogenic activities (3). Although many studies have focused on the sediments and zoobenthos as traditional indicators of pollution and fish habitat preferences factors (4-6), data regarding connections between water microbiota and ichthyofauna diversity of the Danube river remains scarce. As in all natural habitats, different trophic communities are linked together 
tightly and although some may seem unrelated, the causality effects are always present. Current studies between gut microbiota and health in humans have raised the same questions in the aquatic ecotoxicology field, where a strong link between gut microbiota and fish health began to take shape (7-9). No matter the species, the gastrointestinal tract is the primary site of interaction between the host immune system and microorganisms, both symbiotic and pathogenic, resulting in the wellness and fitness of the specimen.

The fish intestinal tract is considered to be the main portal for pathogens $(10,11)$ and as environmental and ecological factors shape the intestinal tract microbiota (12, 13) a correlation between water microbiota and fish diversity is thus consequential. Another factor worth mentioning is that small variations in water $\mathrm{pH}$ are also a factor in fish communication and social interaction patterns (14), having important effects on the growth and reproduction of the fish assemblages (15-17) and as well as on bacterial community composition $(8,18)$.

Variation in water microbiome patterns have been reported $(19,20)$ as well as their distinct potential functions of nitrogen and sulfur metabolisms that lead to changes in water quality and sediment properties. The variation of bacterial communities, which regulate the core biogeochemical processes such as carbon and nitrogen metabolisms in aquatic ecosystems (21), indirectly influences ichthyofauna. Nitrogen fixing bacteria that convert nitrite into the much safer for fish nitrate have a crucial role in fish habitats but also in their physiology, recent studies revealing a symbiotic relationship between fish gills and several ammonia oxidizing and denitrifying bacteria (22). However, not all bacteria act in a beneficial or even near symbiotic manner with fish specimens or fish communities. Coliforms, which are generally considered harmless water pollution indicators, can affect fish gills health (23) as well as transfer drug resistance to pathogens with detrimental consequences for both humans and fish (23-26).

\section{Materials and Methods}

\subsection{Sampling Sites and Methodological Approach}

Along the Danube sector, 11 locations were chosen measuring a total river length of $401 \mathrm{~km}$ (Fig. 1), from which two are the confluence of the Danube river with its tributaries (locations 5 and 6). Water physico-chemical parameters such as general hardness, carbonate hardness, redox, conductivity, temperature, as well as $\mathrm{pH}$, carbon dioxide, dissolved oxygen and oxygen saturation were measured in situ using a portable multi-parameter analyzer (HQ40D Hach-Lange, UK). All physico-chemical sampling took place around 10-11 a.m. hours, as the period of the day just like the temperature, may influence water oxygen saturation levels.

Ichthyofauna sampling was performed with the use of the non-lethal electrofishing devices (SAMUS 725 MP and SUM Electrofisher, Poland), calipers and scales were used to measure the length and weight of the captured specimens. All field meters and equipment were verified and calibrated according to the manufacturer's instructions and specifications prior to use.

Sterile $120 \mathrm{~mL}$ containers and sterile brown $50 \mathrm{~mL}$ Falcon tubes were used to collect surface water, samples corresponding to the 11 locations (S1-S11) were stored and transported at $4^{\circ} \mathrm{C}$ within 24 hours for microbiological analysis. 


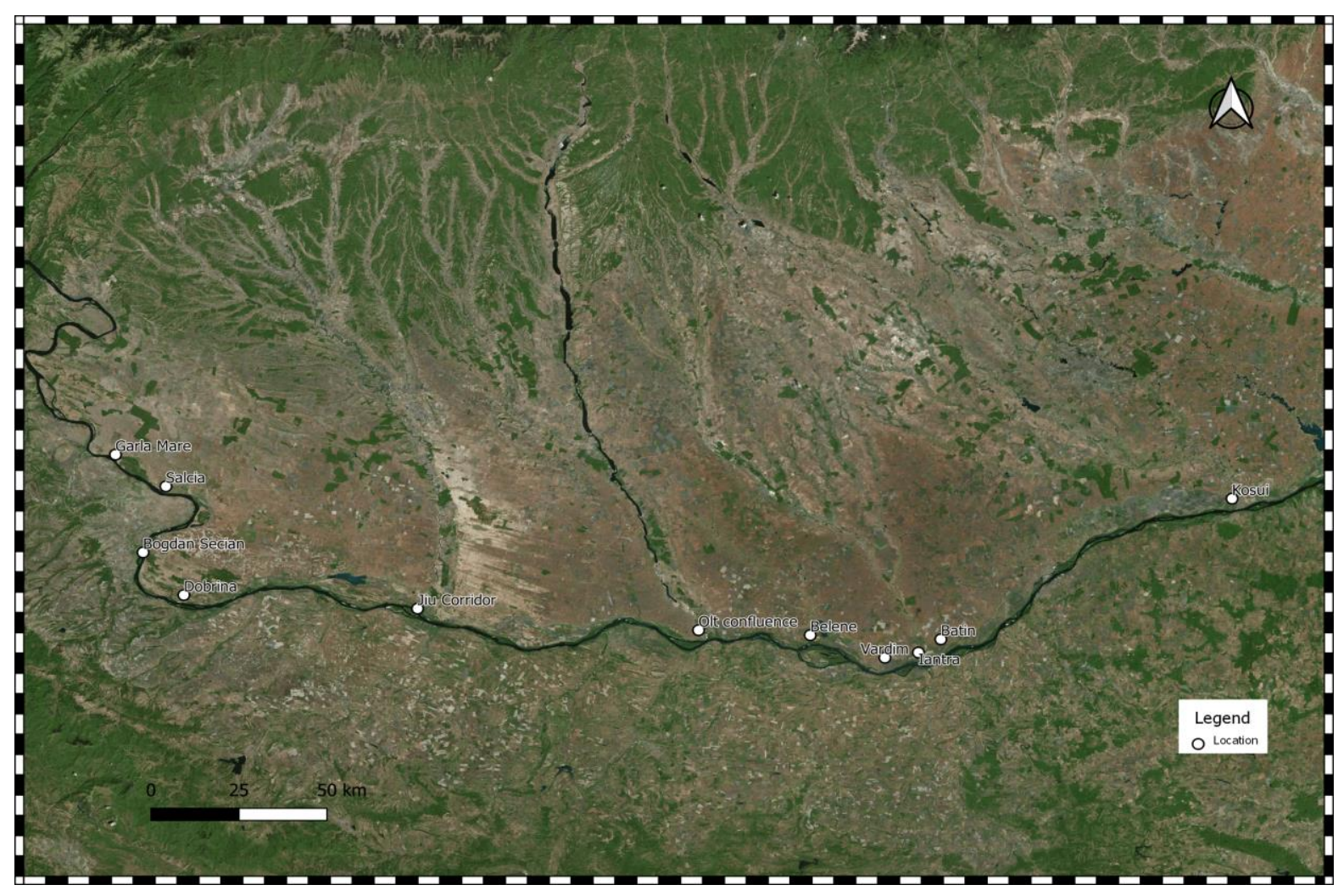

Figure 1. Satellite image of the Danube Romanian-Bulgarian sector, 11 locations (1Garla Mare; 2-Salcia; 3-Bogdan Secian; 4-Dobrina; 5-Jiu Corridor; 6-Olt Confluence; 7Belene; 8-Vardim; 9-Iantra; 10-Batin; 11-Kosui) measuring a total river length of 401 $\mathrm{km}$.

\subsection{Water Physico-Chemical Analysis}

Apart from the in situ water probing, chemical analysis colorimetric kits (Merck Aquaquant, Darmstadt, Germany) were used to evaluate the levels of ammonium, nitrogen dioxide, nitrate, phosphate, silicon dioxide, iron and cooper. The robustness and efficiency of the Merck colorimetric tests being based on the reaction between two chemicals (reagent $A$ and reagent $B$ ) with a certain amount of water sample pipetted in the designated test tubes, accordingly to each kit specifications

\subsection{Water Microbiology Analysis}

Water samples were filtrated through sterile $0.45 \mu \mathrm{m}$ white gridded cellulose membranes with the use of a filtration unit (Merck Eazy-Fit, Darmstadt, Germany), 100 $\mathrm{mL}$ volumes were used to perform the assays. The cellulose membranes were then placed on petri plates with chromogenic agar media (CCA) and on petri plates with Slanetz Bartley media (SB). Confirmation of SB media results was performed with the use of bile esculin azide agar, 2 hours after incubation at $44^{\circ} \mathrm{C}$, accordingly to ISO 7899-2 guidelines (27). The esculin from the bile esculin azide media is hydrolyzed by the enterococci and Esculetin, the end metabolite, bonds with ferric ions to form a noticeable brown to black compound which diffuses into the medium. Accompanying gram-positive and gram-negative flora is inhibited by the azide anion and bile salts, thus reaching a selectivity for enterococci.

Modified Postgate media was used to determine sulphate reducing bacteria (SRB). SRB are anaerobic microorganisms that use sulphate as a terminal electron acceptor, playing a key role in the sulfur and carbon cycles. The determination of SRB densities was performed with the use of serial dilutions. In brief, 9 tubes with Postgate media were used per sample as in: 3 tubes containing $1 \mathrm{~mL}$ of sample, 3 tubes with a $10^{-1}$ dilution and another 3 tube with a $10^{-2}$ dilution. All 99 test tubes were incubated for 7 
days at $37^{\circ} \mathrm{C}$ and then checked for the presence of ferrous sulphate, a black precipitate that indicates the presence of SRB, afterwards the microorganism number (MO) and most probable number (MPN) were calculated.

For total heterotrophs determinations, $1 \mathrm{~mL}$ of sample as well as $10^{-1}, 10^{-2}$ and $10^{-}$ 3 dilutions from each sample were placed in petri dishes and agar-agar media heated at $45^{\circ} \mathrm{C}$ was poured over the sample and then homogenized. The sample containing media was left to cool and was then incubated for $48 \mathrm{~h}$ at $22{ }^{\circ} \mathrm{C}$, after incubation the colonies were counted.

\subsection{Ichthyofauna Diversity Determination}

Electrofishing was performed in linear $500 \mathrm{~m}$ transects, upstream and downstream over shore, as well as over the main water body, with a focus on accurate identification of the inhabitant fish species. Numbed specimens were collected with telescopic pond nets and then classified, weighted and measured, before gently being released back into the water stream. Composition, diversity, abundance and the degree of dominance were noted.

\subsection{Geographic Information System (GIS) Mapping}

Geographic Information Systems (GIS) integrates both hardware and software as well as data for capturing and analyzing all referenced geographic information, allowing the user to visualize and interpret data in many ways. It can be described as a database management system which usually presents the data to the user in an interactive graphical way that can be queried and analyzed. Thus, a correlation between relationships, patterns and trends is made possible in the form of maps, reports, or graphs. Data collection for the 11 locations was performed using a dedicated GPS device (Garmin, U.S.A), the coordinates were processed and entered in the GIS freeware software Qgis and Saga, having as a base map the freeware source from Bing search engine, the measurement error of the location being less than one meter.

\section{Results and Discussions}

\subsection{Water Analysis}

Physico-chemical and microbiological results from the 11 locations (Tables 1, 2) reveal the quality of water in the sector of interest, with variations linked to the anthropogenic activities.

Although the microbiological, physical and chemical parameters were found to be in what are considered normal ranges (28-30), locations 3 (Bogdan Secian), 7 (Belene), 8 (Vardim) and 11 (Kosui) are the most affected from a microbiological point of view, as they are subject to wastewater impact from the residential settlements and from the Bulgarian penitentiary located on Belene Island.

Table 1

Physico-chemical measurements

\begin{tabular}{cccccc}
\hline Location & \multicolumn{2}{c}{ Physical parameters } & \multicolumn{2}{c}{ Chemical parameters } \\
\hline & conductivity & $204 \mu \mathrm{S} / \mathrm{cm}$ & $\mathrm{KH}=$ & 8 & $\mathrm{dKH}$ \\
\cline { 2 - 6 } & $\mathrm{pH}$ & 8,07 & $\mathrm{GH}=$ & 10 & $\mathrm{dGH}$ \\
\cline { 2 - 6 } Garla Mare (S1) & redox & $-60,60 \mathrm{mV}$ & $\mathrm{CO}_{2}=$ & $<15$ & $\mathrm{mg} / \mathrm{L}$ \\
\cline { 2 - 6 } & temperature & $11,8^{\circ} \mathrm{C}$ & $\mathrm{NH}_{4}=$ & $<0,05$ & $\mathrm{mg} / \mathrm{L}$ \\
\cline { 2 - 6 } & dissolved oxygen & $9,24 \mathrm{mg} / \mathrm{L}$ & $\mathrm{NO}_{2}=$ & 0,25 & $\mathrm{mg} / \mathrm{L}$ \\
\cline { 2 - 6 } & oxygen saturation & $83,50 \%$ & $\mathrm{NO}_{3}=$ & 25 & $\mathrm{mg} / \mathrm{L}$ \\
\hline
\end{tabular}


Salcia (S2)

Bogdan Secian (S3)

Jiu Corridor (S5)

Olt Confluence (S6)

Belene (S7)

conductivity $\mathrm{pH}$
$\mathrm{PO}_{4}=$

$\mathrm{SiO}_{2}=$

$\mathrm{Fe}=$

$\mathrm{Cu}=$

$\mathrm{KH}=$

$\mathrm{GH}=$

$\mathrm{CO}_{2}=$

$-51,80 \mathrm{mV}$

$13,9^{\circ} \mathrm{C}$

temperature

$8,94 \mathrm{mg} / \mathrm{L}$

$\mathrm{NH}_{4}=$

$83,40 \%$

$\mathrm{NO}_{2}=$

$\mathrm{NO}_{3}=$
$\mathrm{PO}_{4}=$

$\mathrm{SiO}_{2}=$

$\mathrm{Fe}=$

$\mathrm{Cu}=$

$\mathrm{KH}=$

$\mathrm{GH}=$

redox

temperature

dissolved oxygen

oxygen saturation

conductivity $\mathrm{pH}$ redox

temperature

dissolved oxygen

oxygen saturation
$324 \mu \mathrm{S} / \mathrm{cm}$

8,09

$-61,70 \mathrm{mV}$

$12,5^{\circ} \mathrm{C}$

$9,33 \mathrm{mg} / \mathrm{L}$

$86,10 \%$
$\mathrm{CO}_{2}=$

$\mathrm{NH}_{4}=$

$\mathrm{NO}_{2}=$

$\mathrm{NO}_{3}=$

$\mathrm{PO}_{4}=$

$\mathrm{SiO}_{2}=$

$\mathrm{Fe}=$

$\mathrm{Cu}=$

$\mathrm{KH}=$

$\mathrm{GH}=$

$\mathrm{CO}_{2}=$

$-60,50 \mathrm{mV}$

$\mathrm{NH}_{4}=$

$13,4^{\circ} \mathrm{C}$

$\mathrm{NO}_{2}=$

$9,02 \mathrm{mg} / \mathrm{L}$

$\mathrm{NO}_{3}=$

$\mathrm{PO}_{4}=$

$\mathrm{SiO}_{2}=$

$\mathrm{Fe}=$

$\mathrm{Cu}=$

$<0,05$

$>6,0$

$\mathrm{mg} / \mathrm{L}$

$<0,02$

$\mathrm{mg} / \mathrm{L}$

$<0,1$

8

$\mathrm{mg} / \mathrm{L}$

$10 \quad \mathrm{dGH}$

$<15$

$<0,05$

$\mathrm{mg} / \mathrm{L}$

0,25

25

$<0,05$

$\mathrm{mg} / \mathrm{L}$

$>6,0$

$<0,02$

$\mathrm{mg} / \mathrm{L}$

$\mathrm{mg} / \mathrm{L}$

$\mathrm{mg} / \mathrm{L}$

$<0,1$

8

8

15

$<0,05$

$\mathrm{mg} / \mathrm{L}$

$\mathrm{KH}=$

0,25

$\mathrm{mg} / \mathrm{L}$

Jiu Corridor (S5)

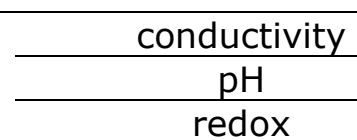

temperature dissolved oxygen oxygen saturation

$207 \mu \mathrm{S} / \mathrm{cm}$
8,36
$-76,60 \mathrm{mV}$
$11,0^{\circ} \mathrm{C}$
$10,87 \mathrm{mg} / \mathrm{L}$
$97,10 \%$

$\mathrm{GH}=$

$\mathrm{CO}_{2}=$

$\mathrm{NH}_{4}=$

$\mathrm{NO}_{2}=$

$\mathrm{NO}_{3}=$

$\mathrm{PO}_{4}=$

$\mathrm{SiO}_{2}=$

$\mathrm{Fe}=$

$\mathrm{Cu}=$

$\mathrm{KH}=$

$\mathrm{GH}=$

$\mathrm{CO}_{2}=$

$\mathrm{NH}_{4}=$

$-65,90 \mathrm{mV}$

temperature

$10,8^{\circ} \mathrm{C}$

dissolved oxygen

oxygen saturation

$89,80 \%$

$\mathrm{NO}_{2}=$

$\mathrm{NO}_{3}=$

$\mathrm{PO}_{4}=$

$\mathrm{SiO}_{2}=$

$\mathrm{Fe}=$

$\mathrm{Cu}=$

$\mathrm{KH}=$

$\mathrm{GH}=$

$55 \mu \mathrm{S} / \mathrm{cm}$

8,16

\begin{abstract}
GH
\end{abstract}
$\mathrm{mg} / \mathrm{L}$

$\mathrm{dKH}$

$\mathrm{dGH}$

$\mathrm{mg} / \mathrm{L}$

$\mathrm{mg} / \mathrm{L}$

$\mathrm{mg} / \mathrm{L}$

\begin{tabular}{cc}
40 & $\mathrm{mg} / \mathrm{L}$ \\
$<0,05$ & $\mathrm{mg} / \mathrm{L}$ \\
\hline$>6,0$ & $\mathrm{mg} / \mathrm{L}$
\end{tabular}

$>6,0 \mathrm{mg} / \mathrm{L}$

$<0,02 \quad \mathrm{mg} / \mathrm{L}$

$<0,1$

8 dKH

$\mathrm{mg} / \mathrm{L}$

10 dGH

$15 \mathrm{mg} / \mathrm{L}$

$<0,05 \mathrm{mg} / \mathrm{L}$

$0,25 \mathrm{mg} / \mathrm{L}$

$25 \mathrm{mg} / \mathrm{L}$

$<0,02 \quad \mathrm{mg} / \mathrm{L}$

$\begin{array}{cc}>6,0 & \mathrm{mg} / \mathrm{L} \\ <0,02 & \mathrm{mg} / \mathrm{L}\end{array}$

$<0,02$

$\mathrm{mg} / \mathrm{L}$

$\begin{array}{cc}<0,1 & \mathrm{mg} / \mathrm{L} \\ 4,5 & \mathrm{dKH}\end{array}$

$>7$

$\mathrm{dKH}$

$>7 \mathrm{dGH}$

\begin{tabular}{ll}
$<0,05$ & $\mathrm{mg} / \mathrm{L}$ \\
\hline & $\mathrm{mg} / \mathrm{L}$
\end{tabular}

$0,5 \mathrm{mg} / \mathrm{L}$

$25 \mathrm{mg} / \mathrm{L}$

$<0,05 \mathrm{mg} / \mathrm{L}$

$>6,0 \mathrm{mg} / \mathrm{L}$

$<0,02 \quad \mathrm{mg} / \mathrm{L}$

$<0,1 \quad \mathrm{mg} / \mathrm{L}$

$8 \quad \mathrm{dKH}$

$>14 \mathrm{dGH}$

$20 \mathrm{mg} / \mathrm{L}$

$<0,05 \mathrm{mg} / \mathrm{L}$

$0,5 \mathrm{mg} / \mathrm{L}$

$40 \quad \mathrm{mg} / \mathrm{L}$

$<0,02 \mathrm{mg} / \mathrm{L}$

$>6,0 \mathrm{mg} / \mathrm{L}$

$<0,02 \quad \mathrm{mg} / \mathrm{L}$

$<0,1 \mathrm{mg} / \mathrm{L}$

$\begin{array}{cc}8 & \mathrm{dKH} \\ >10 & \mathrm{dGH}\end{array}$




\begin{tabular}{|c|c|c|c|c|c|}
\hline & redox & $-65,50 \mathrm{mV}$ & $\mathrm{CO}_{2}=$ & 15 & $\mathrm{mg} / \mathrm{L}$ \\
\hline & temperature & $11,4^{\circ} \mathrm{C}$ & $\mathrm{NH}_{4}=$ & $<0,05$ & $\mathrm{mg} / \mathrm{L}$ \\
\hline & dissolved oxygen & $9,66 \mathrm{mg} / \mathrm{L}$ & $\mathrm{NO}_{2}=$ & 0,5 & $\mathrm{mg} / \mathrm{L}$ \\
\hline & oxygen saturation & $87,20 \%$ & $\mathrm{NO}_{3}=$ & 40,00 & $\mathrm{mg} / \mathrm{L}$ \\
\hline & & & $\mathrm{PO}_{4}=$ & $<0,02$ & $\mathrm{mg} / \mathrm{L}$ \\
\hline & & & $\mathrm{SiO}_{2}=$ & $>6,0$ & $\mathrm{mg} / \mathrm{L}$ \\
\hline & & & $\mathrm{Fe}=$ & $<0,02$ & $\mathrm{mg} / \mathrm{L}$ \\
\hline & & & $\mathrm{Cu}=$ & $<0,1$ & $\mathrm{mg} / \mathrm{L}$ \\
\hline \multirow{10}{*}{ Vardim (S8) } & conductivity & $227 \mu \mathrm{S} / \mathrm{cm}$ & $\mathrm{KH}=$ & 10 & $\mathrm{dKH}$ \\
\hline & $\mathrm{pH}$ & 8,18 & $\mathrm{GH}=$ & $>14$ & $\mathrm{dGH}$ \\
\hline & redox & $-65,60 \mathrm{mV}$ & $\mathrm{CO}_{2}=$ & 30 & $\mathrm{mg} / \mathrm{L}$ \\
\hline & temperature & $11,2^{\circ} \mathrm{C}$ & $\mathrm{NH}_{4}=$ & $<0,05$ & $\mathrm{mg} / \mathrm{L}$ \\
\hline & dissolved oxygen & $10,36 \mathrm{mg} / \mathrm{L}$ & $\mathrm{NO}_{2}=$ & 0,5 & $\mathrm{mg} / \mathrm{L}$ \\
\hline & oxygen saturation & $87,70 \%$ & $\mathrm{NO}_{3}=$ & 40 & $\mathrm{mg} / \mathrm{L}$ \\
\hline & & & $\mathrm{PO}_{4}=$ & $<0,02$ & $\mathrm{mg} / \mathrm{L}$ \\
\hline & & & $\mathrm{SiO}_{2}=$ & $>6,0$ & $\mathrm{mg} / \mathrm{L}$ \\
\hline & & & $\mathrm{Fe}=$ & $<0,02$ & $\mathrm{mg} / \mathrm{L}$ \\
\hline & & & $\mathrm{Cu}=$ & $<0,1$ & $\mathrm{mg} / \mathrm{L}$ \\
\hline \multirow{10}{*}{ Iantra (S9) } & conductivity & $262 \mu \mathrm{S} / \mathrm{cm}$ & $\mathrm{KH}=$ & 6 & $\mathrm{dKH}$ \\
\hline & $\mathrm{pH}$ & 8,22 & $\mathrm{GH}=$ & $>10$ & $\mathrm{dGH}$ \\
\hline & redox & $-68,70 \mathrm{mV}$ & $\mathrm{CO}_{2}=$ & $<15$ & $\mathrm{mg} / \mathrm{L}$ \\
\hline & temperature & $10,6^{\circ} \mathrm{C}$ & $\mathrm{NH}_{4}=$ & $<0,05$ & $\mathrm{mg} / \mathrm{L}$ \\
\hline & dissolved oxygen & $10,16 \mathrm{mg} / \mathrm{L}$ & $\mathrm{NO}_{2}=$ & 0,5 & $\mathrm{mg} / \mathrm{L}$ \\
\hline & oxygen saturation & $89,40 \%$ & $\mathrm{NO}_{3}=$ & 40,00 & $\mathrm{mg} / \mathrm{L}$ \\
\hline & & & $\mathrm{PO}_{4}=$ & $<0,02$ & $\mathrm{mg} / \mathrm{L}$ \\
\hline & & & $\mathrm{SiO}_{2}=$ & $>6,0$ & $\mathrm{mg} / \mathrm{L}$ \\
\hline & & & $\mathrm{Fe}=$ & $<0,02$ & $\mathrm{mg} / \mathrm{L}$ \\
\hline & & & $\mathrm{Cu}=$ & $<0,1$ & $\mathrm{mg} / \mathrm{L}$ \\
\hline \multirow{10}{*}{ Batin (S10) } & conductivity & $243 \mu \mathrm{S} / \mathrm{cm}$ & $\mathrm{KH}=$ & 10 & $\mathrm{dKH}$ \\
\hline & $\mathrm{pH}$ & 8,15 & $\mathrm{GH}=$ & $>14$ & $\mathrm{dGH}$ \\
\hline & redox & $-65,00 \mathrm{mV}$ & $\mathrm{CO}_{2}=$ & $<15$ & $\mathrm{mg} / \mathrm{L}$ \\
\hline & temperature & $11,2^{\circ} \mathrm{C}$ & $\mathrm{NH}_{4}=$ & $<0,05$ & $\mathrm{mg} / \mathrm{L}$ \\
\hline & dissolved oxygen & $10,55 \mathrm{mg} / \mathrm{L}$ & $\mathrm{NO}_{2}=$ & 0,5 & $\mathrm{mg} / \mathrm{L}$ \\
\hline & oxygen saturation & $93,80 \%$ & $\mathrm{NO}_{3}=$ & 40 & $\mathrm{mg} / \mathrm{L}$ \\
\hline & & & $\mathrm{PO}_{4}=$ & $<0,02$ & $\mathrm{mg} / \mathrm{L}$ \\
\hline & & & $\mathrm{SiO}_{2}=$ & $>6,0$ & $\mathrm{mg} / \mathrm{L}$ \\
\hline & & & $\mathrm{Fe}=$ & $<0,02$ & $\mathrm{mg} / \mathrm{L}$ \\
\hline & & & $\mathrm{Cu}=$ & $<0,1$ & $\mathrm{mg} / \mathrm{L}$ \\
\hline \multirow{10}{*}{ Kosui (S11) } & conductivity & $253 \mu \mathrm{S} / \mathrm{cm}$ & $\mathrm{KH}=$ & 10 & $\mathrm{dKH}$ \\
\hline & $\mathrm{pH}$ & 8,15 & $\mathrm{GH}=$ & $>14$ & $\mathrm{dGH}$ \\
\hline & redox & $-65,00 \mathrm{mV}$ & $\mathrm{CO}_{2}=$ & 15 & $\mathrm{mg} / \mathrm{L}$ \\
\hline & temperature & $11,7^{\circ} \mathrm{C}$ & $\mathrm{NH}_{4}=$ & $<0,05$ & $\mathrm{mg} / \mathrm{L}$ \\
\hline & dissolved oxygen & $9,80 \mathrm{mg} / \mathrm{L}$ & $\mathrm{NO}_{2}=$ & 1,0 & $\mathrm{mg} / \mathrm{L}$ \\
\hline & oxygen saturation & $88,40 \%$ & $\mathrm{NO}_{3}=$ & 50 & $\mathrm{mg} / \mathrm{L}$ \\
\hline & & & $\mathrm{PO}_{4}=$ & $<0,02$ & $\mathrm{mg} / \mathrm{L}$ \\
\hline & & & $\mathrm{SiO}_{2}=$ & $>6,0$ & $\mathrm{mg} / \mathrm{L}$ \\
\hline & & & $\mathrm{Fe}=$ & $<0,02$ & $\mathrm{mg} / \mathrm{L}$ \\
\hline & & & $\mathrm{Cu}=$ & $<0,1$ & $\mathrm{mg} / \mathrm{L}$ \\
\hline
\end{tabular}

Coliforms such as Escherichia Coli (E. coli) and other coliforms were found in significant densities, represented as colony forming units (CFU), in S3, S7, S8 and S10, all locations being residential and agricultural areas. Without a strong relation between water physico-chemical parameters and coliforms as well as fecal coliform 
concentrations, we suggest that the high E. coli and other Coliforms densities are due to both human and animal fecal dejection and especially a consequence of the practice of manure spreading as agricultural fertilizer, which eventually end up in the main water stream during rainfall.

The Enterococcus bacteria, a known indicator of the presence of fecal materia in water, was found most prominent in S7 followed by S11 and S3. High density of Enterrococcus in S7 can be directly linked with the presence of Belene penitenciary located on Belene island. The lack of evidence of Enterrococus in S8 suggests that the contamination in Location 8 is not due to fecal matter, however, further investigations should be made regarding this topic.

Heterotrophic bacteria represent the totality of bacteria that requires organic nutrients for growth, thus it cannot be used as an indicator of fecal contamination. Heterotrophic plate count technique (HPC) includes a wide range of bacterial genera, which may include primary and secondary pathogens concluded as total viable count (TVC). Some bacteria enumerated by HPC method may also include opportunistic pathogens such as Klebsiella, Aeromonas and Pseudomonas, thus the HPC is considered to be a health-based water parameter test (31). The HPC test revealed high concentrations in S10, S8 and S7 with the smallest densities found in S4 and S3, highlighting S7 as a common point of high bacterial density.

Although Sulfate reducing bacteria (SRB) prefer oxygen-deficient environments, samples collected from the surface water, where oxygen saturations were high, shown representative variations, as in: S1, S4, S7, S9-11, with a high density in S11. Anaerobic iron corrosive and key microbiological indicators, SRB (which also generate the smell of rotten egg of hydrogen sulfide) can also be linked with immersed metals suffering biocorrosion $(32,33)$.

Table 2

Microbiological parameters of water samples (CFU/100 mL)

\begin{tabular}{|c|c|c|c|c|c|}
\hline Sample & Coliforms & Escherichia coli & Enterococcus & TVC at $22^{\circ} \mathrm{C}$ & SRB \\
\hline $\mathrm{S} 1$ & $2,3 * 10^{2}$ & 7,3 & 2 & $8,5 * 10^{3}$ & 1 \\
\hline S2 & $1,4 * 10^{2}$ & $1,7 * 10$ & 2 & $3,3 * 10^{3}$ & 0 \\
\hline S3 & $4 * 10^{2}$ & $3 * 10^{2}$ & 8 & $7 * 10^{2}$ & 0 \\
\hline S4 & $1,5 * 10^{2}$ & $1,1 * 10$ & 1 & $6 * 10^{2}$ & 1 \\
\hline S5 & $1,5 * 10^{2}$ & $3,8 * 10$ & 0 & $1 * 10^{3}$ & 0 \\
\hline S6 & $1,6 * 10^{2}$ & $2,4 * 10$ & 2 & $3,2 * 10^{3}$ & 0 \\
\hline S7 & $3,4 * 10^{2}$ & $6,7 * 10$ & $1,7 * 10^{2}$ & $2,9 * 10^{4}$ & 1 \\
\hline S8 & $6,2 * 10$ & $2,5 * 10^{2}$ & 0 & $7,4 * 10^{4}$ & 0 \\
\hline S9 & $1,5 * 10^{2}$ & $1,4 * 10$ & 0 & $1,2 * 10^{4}$ & 1 \\
\hline $\mathrm{S} 10$ & $6,1 * 10^{2}$ & $5,9 * 10^{2}$ & 5 & $8,3 * 10^{4}$ & 2 \\
\hline S11 & $7,5 * 10$ & $7,2 * 10$ & $1,5 * 10$ & $2,2 * 10^{3}$ & $2,5 * 10$ \\
\hline
\end{tabular}

\subsection{Ichthyofauna Diversity}

\subsubsection{Garla Mare Station}

Habitats are maintained at this station by the offshore islands where there is wild vegetation, especially willows that are partially submerged, providing good spawning places for most phytophilic fish species.

The total number of fish species observed at this station was 20 species on shore (Figure 3) water and 12 species in open water (Figure 4). Species of community interest found were Pelecus cultratus and Aspius aspius. 
The dominant fish species at Garla Mare station was Alburnus alburnus, Carassius gibelio and Neogobius fluviatillis, with only one individual observed per species: Blicca bjoerkna, Carassius carassius and Leuciscus idus.

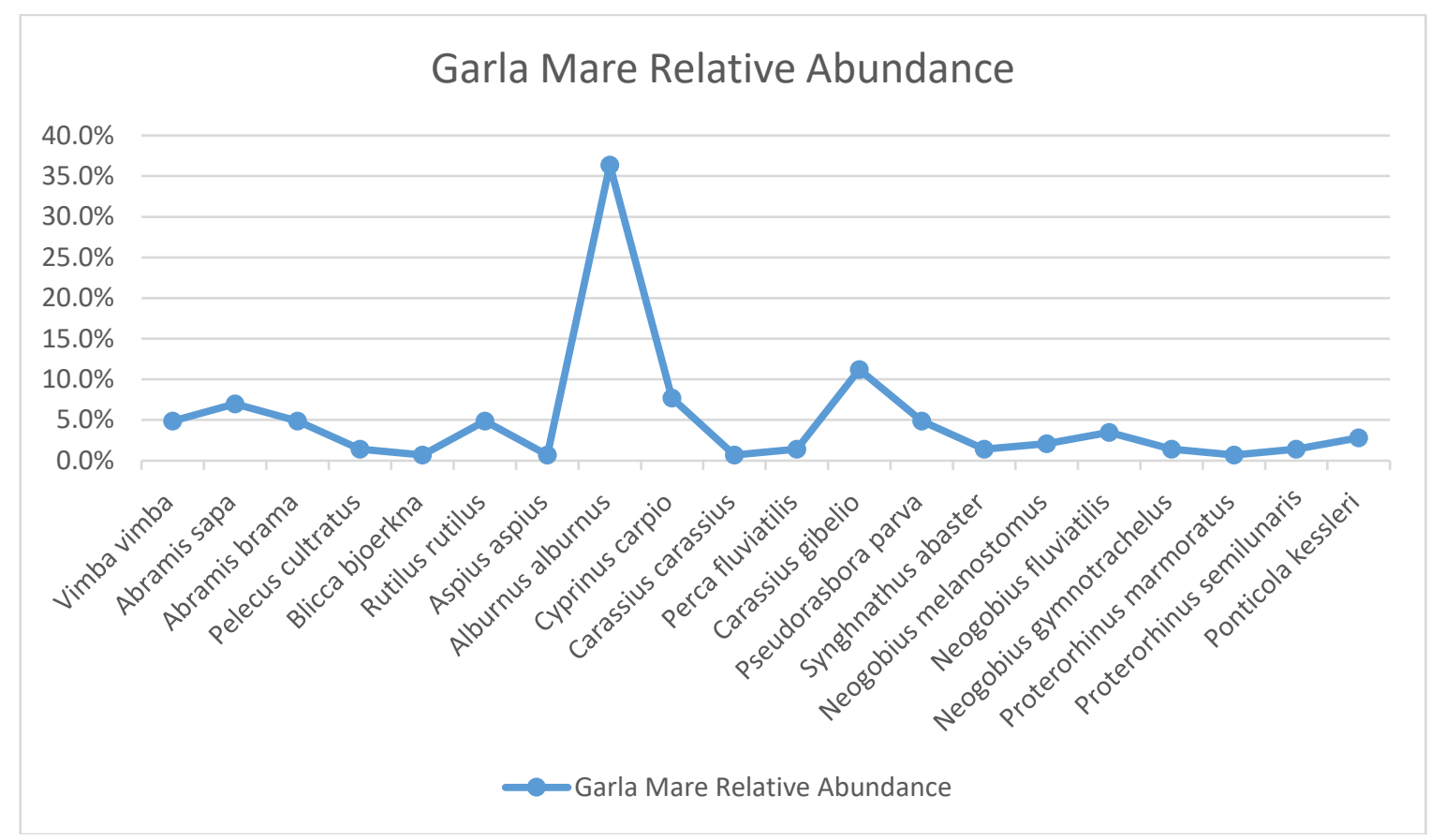

Figure 3. Relative abundance of identified fish species in shore water.

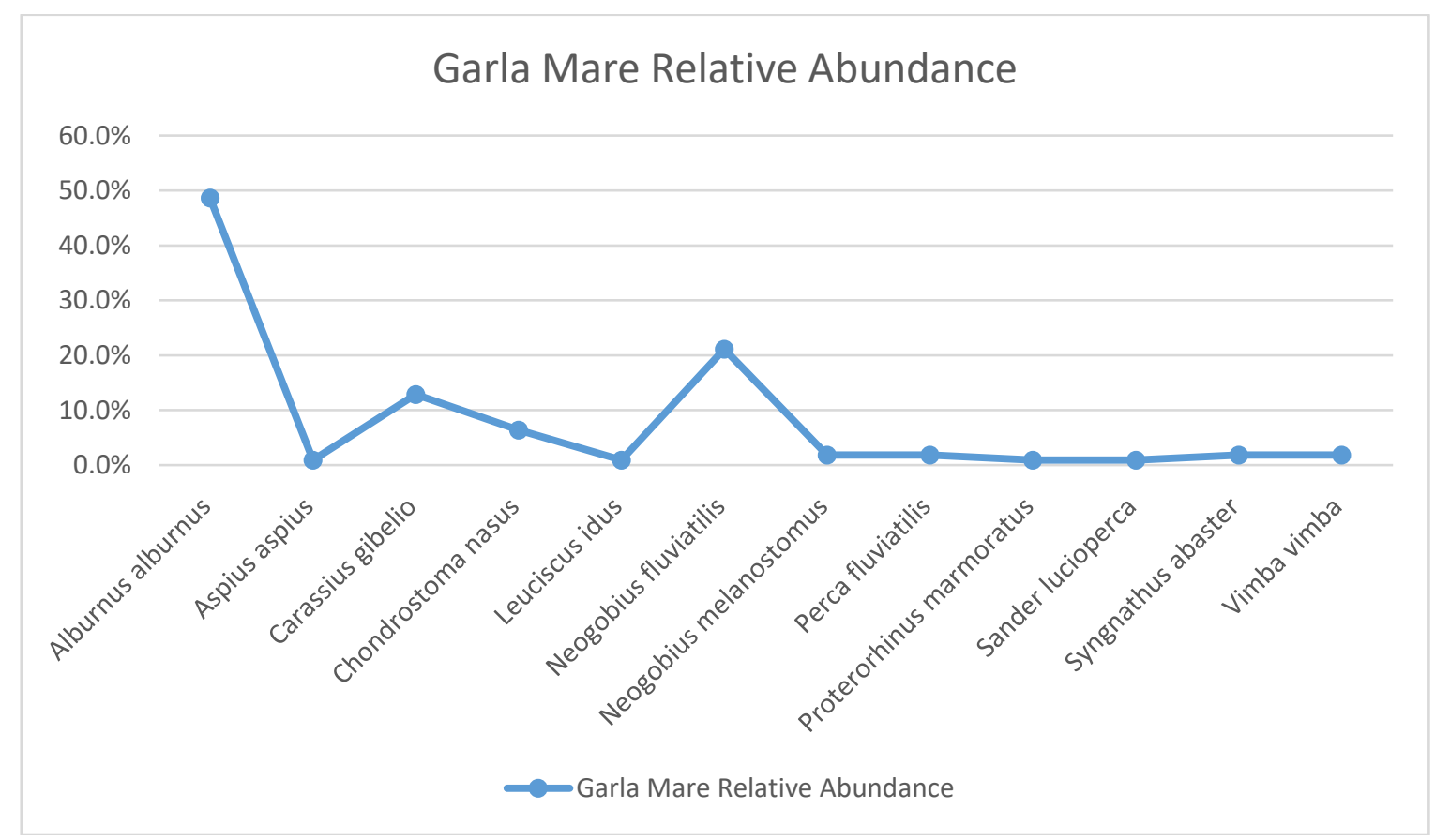

Figure 4. Relative abundance of identified fish species in open water.

\subsubsection{Salcia Station}

For shore water study 5 species were observed (Figure 5) and 8 species were observed in the open water study (Figure 6). No species of community interest was identified. The dominant fish species was: Alburnus alburnus, Carassius gibelio and Neogobius fluviatilis. Species with only one individual observed was Protherinus semilunaris. 


\section{Salcia Relative Abundance}

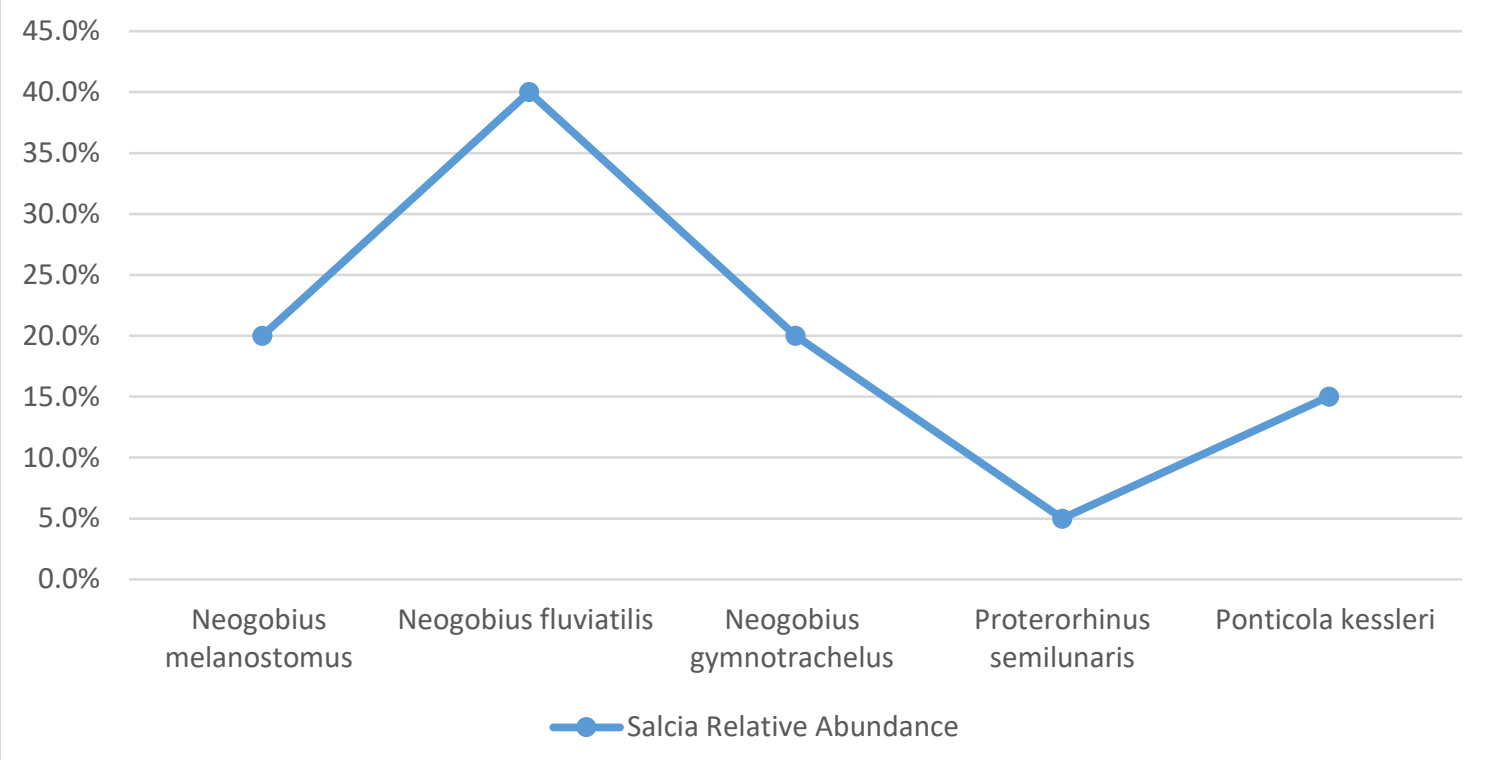

Figure 5. Relative abundance of identified fish species in shore water.

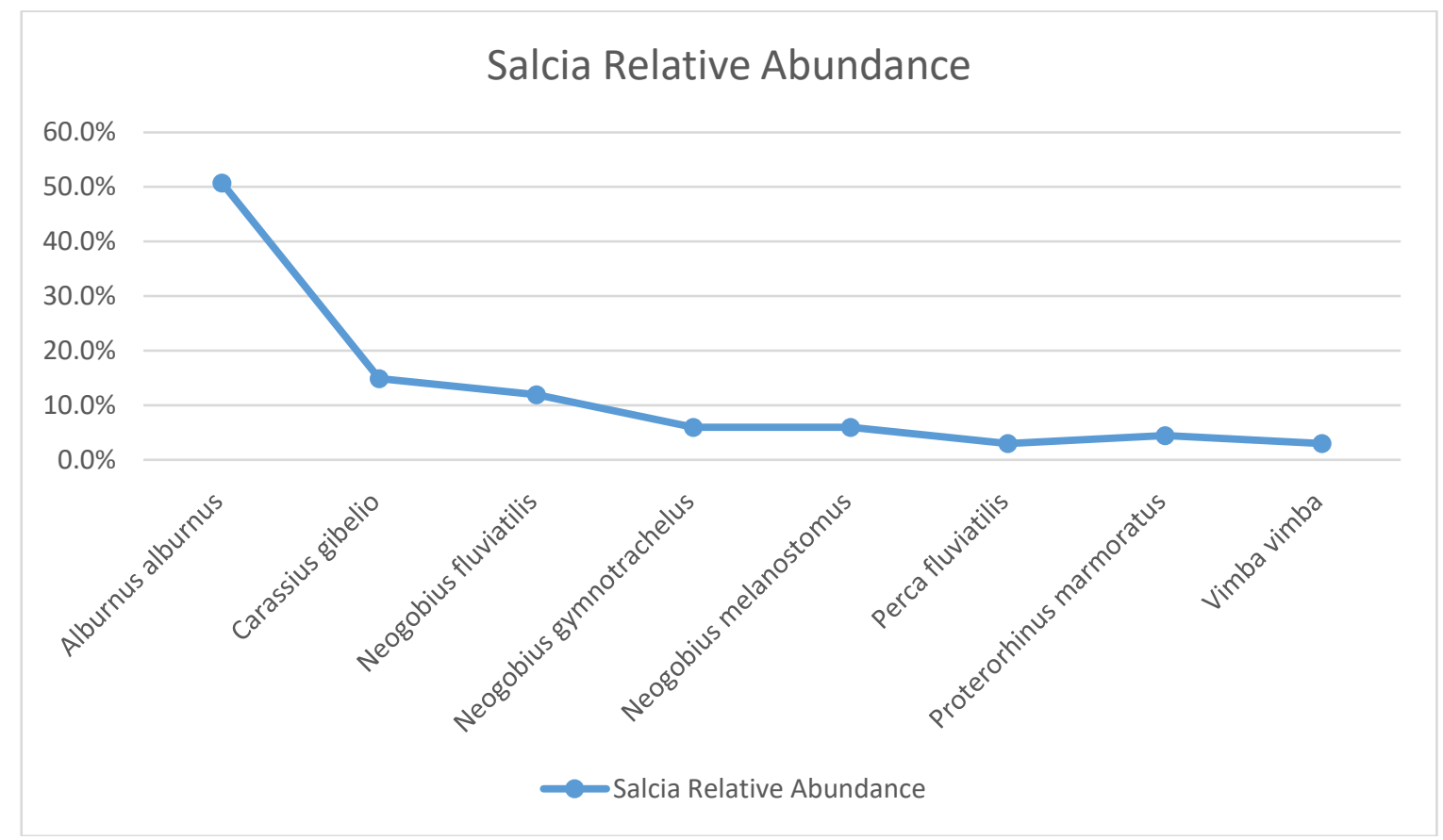

Figure 6. Relative abundance of identified fish species in open water.

\subsubsection{Bogdan Secian Station}

The total number of fish species observed at this station was 4 species on shore water (Figure 7) and 6 species in open water (Figure 8), no species of community interest were found. The dominant fish species at Bogdan Secian station was Alburnus alburnus and Vimba vimba in equal numbers, followed by Neogobius fluviatilis. The less frequent species for this station was Neogobius gymnotrachelus with only two specimens and Neogobius melanostomus with three specimens. 


\section{Bogdan Secian Relative Abundance}

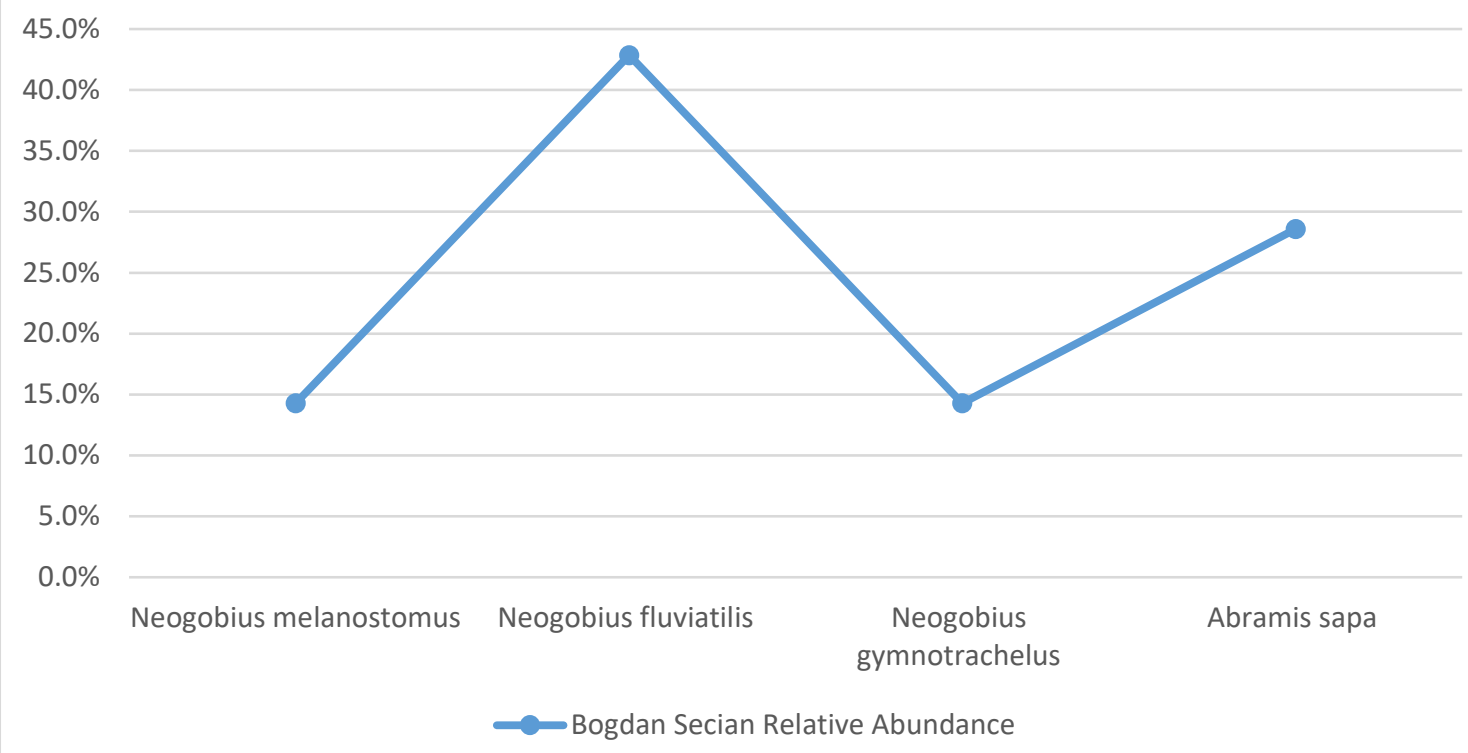

Figure 7. Relative abundance of identified fish species in shore water.

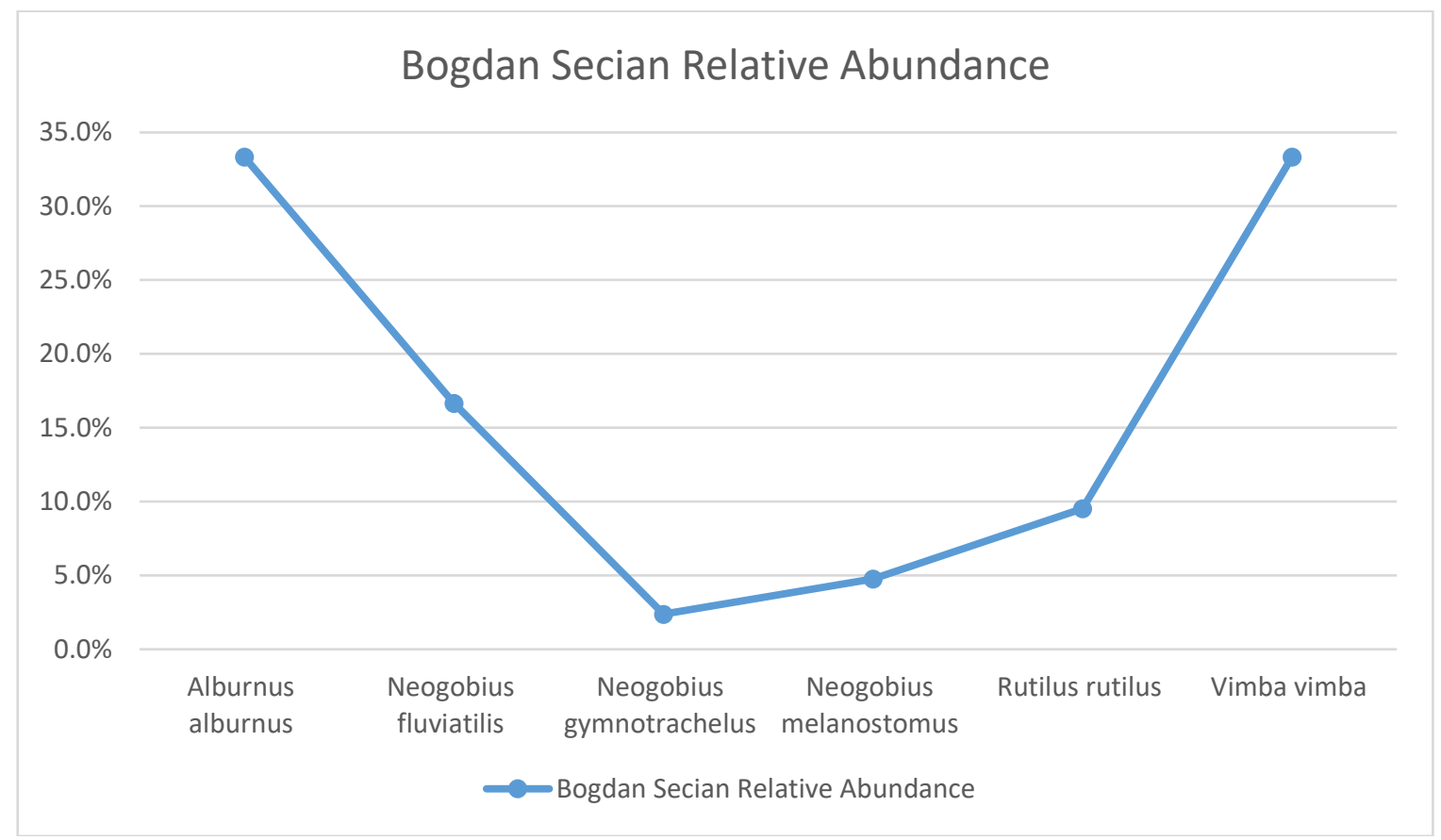

Figure 8. Relative abundance of identified fish species in open water.

\subsubsection{Dobrina Station}

For Dobrina station 4 species were identified in shore water (Figure 9) and 11 species in open water (Figure 10), with 1 species of community interest, Aspius aspius. The dominant fish species were Alburnus alburnus and Neogobius fluviatilis, less frequent species were Blicca bjoerkna and Esox lucius. 


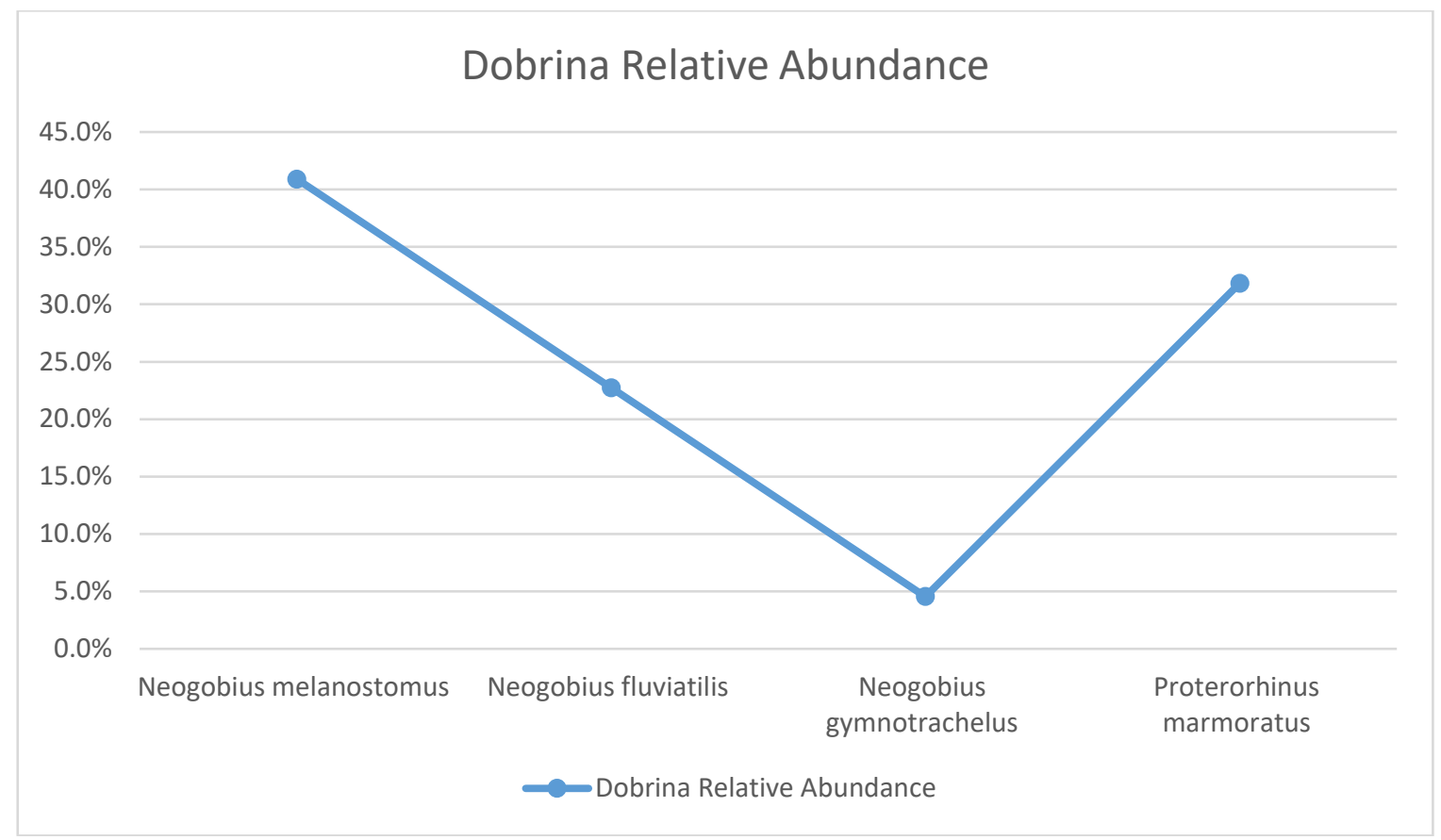

Figure 9. Relative abundance of identified fish species in shore water.

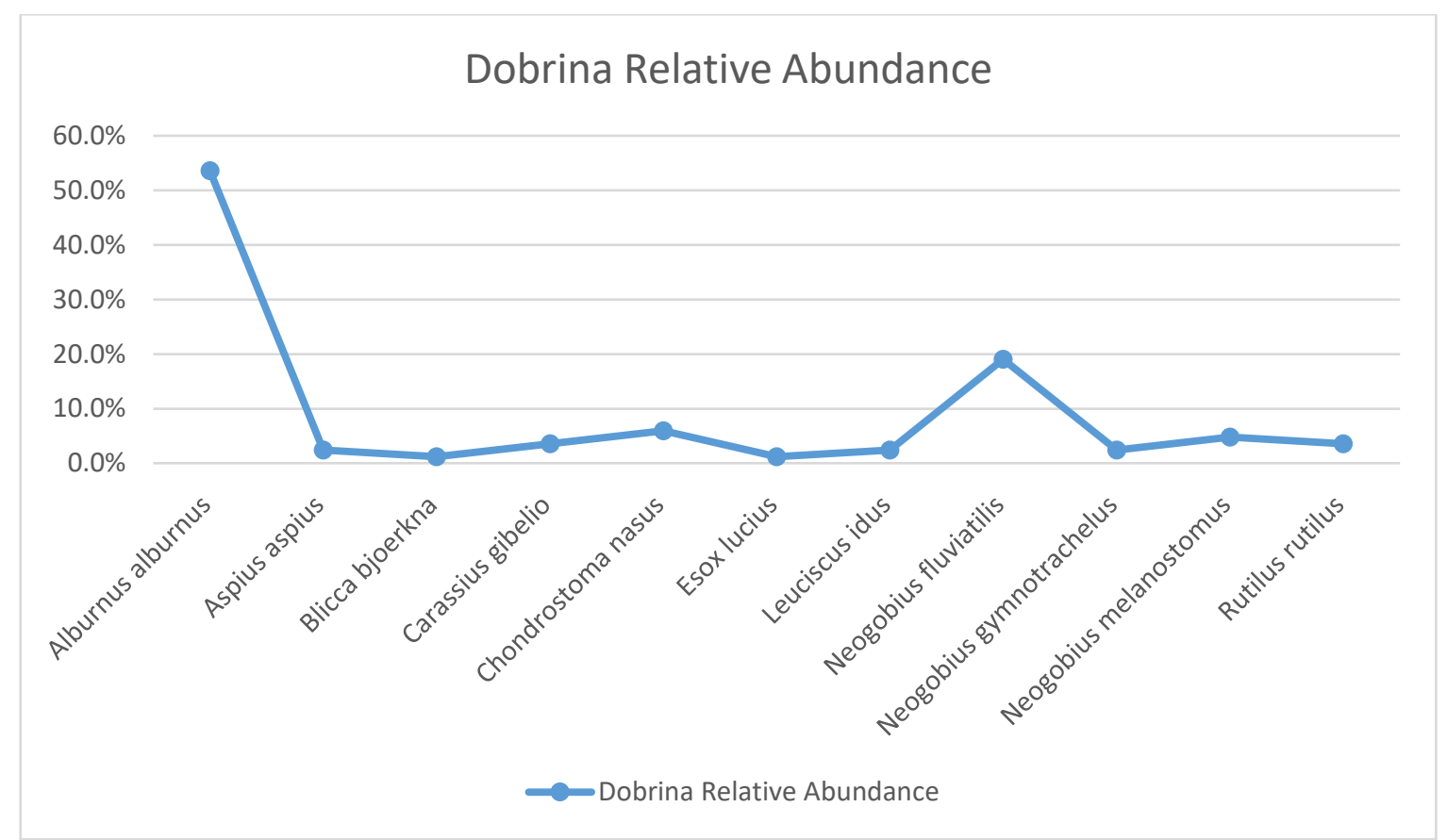

Figure 10. Relative abundance of identified fish species in open water.

\subsubsection{Jiu corridor}

At this confluence point of the Danube with Jiu river, 11 species were found on shore water (Figure 11) and 11 species in open water (Figure 12), with 2 species of community interest: Barbus barbus and Sabajenewia aurata balcanica. The dominant fish species were: Neogobius fluviatilis and Pseudorasbora parva. The less frequent species for this station were: Abramis brama, Sander lucioperca, Leuciscus idus and Sabajenwia aurata balcanica with only one observed individual per species. 


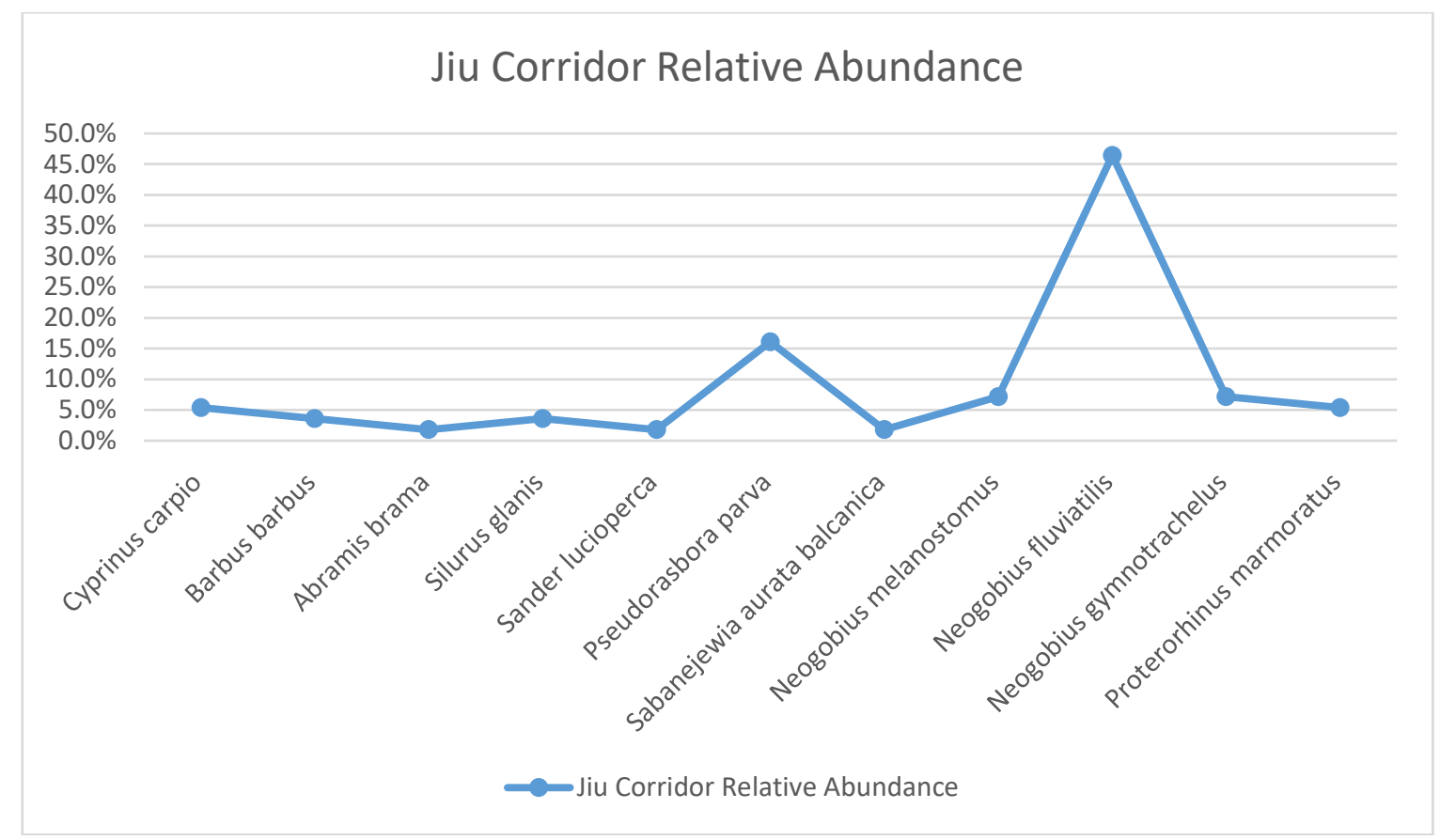

Figure 11. Relative abundance of identified fish species in shore water.

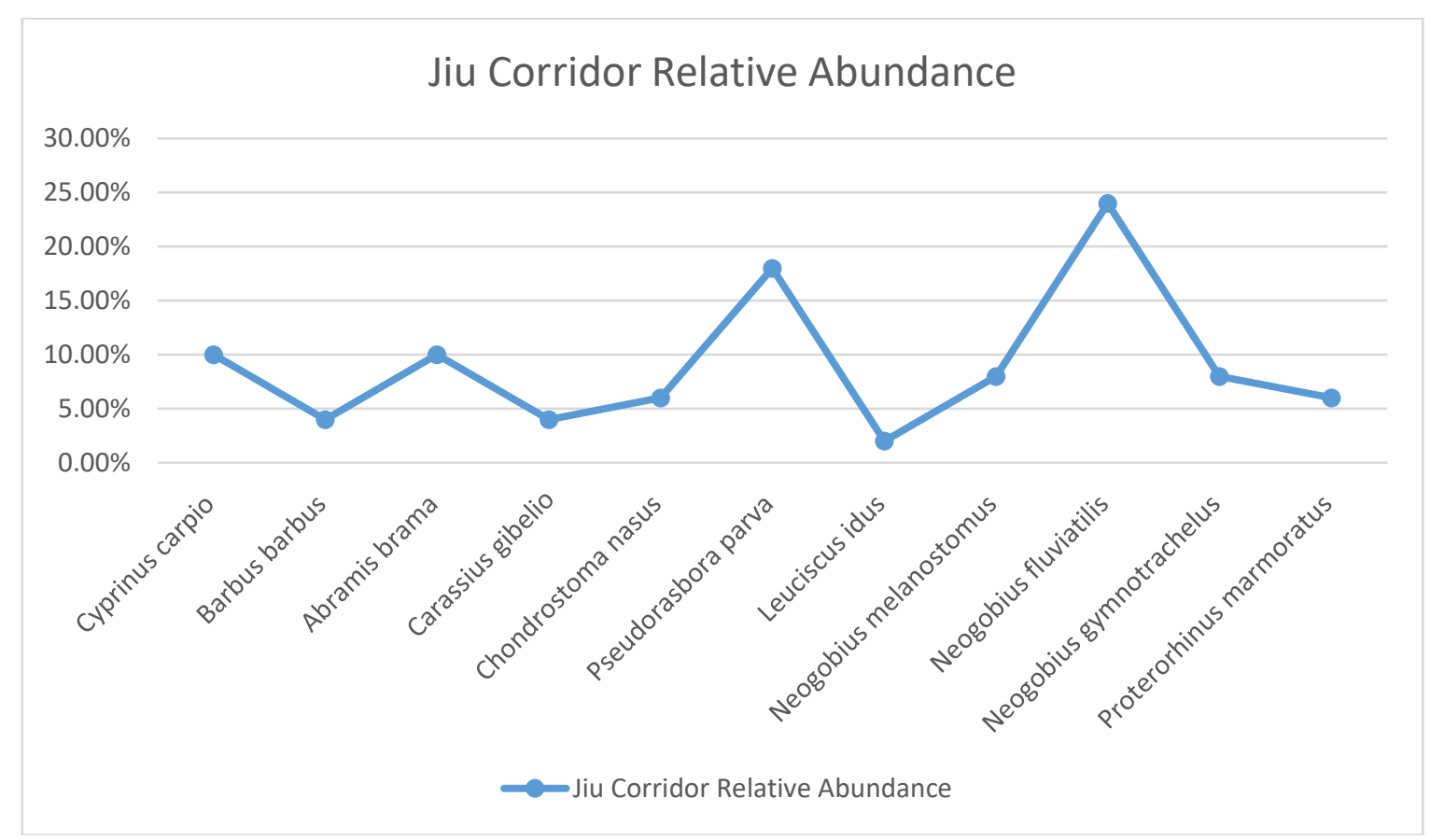

Figure 12. Relative abundance of identified fish species in open water.

\subsubsection{Olt confluence}

The widely spread and invasive species Alburnus alburnus, a lacustrine and fluvial cyprinid, was found to be to dominant at this location both on shore and in the open water. For the shore study a total of 6 species were found and 22 species were identified in the open water study, with 5 species of community interest: Aspius aspius, Barbus barbus, Eudontomyzon mariae, Rhodeus amarus and Sabanejewia aurata romanica. The less frequent species for this station were: Proterorhinus marmoratus, Barbus barbus, Eudontomyzon mariae, Pseudorasbora parva, Sabanejewia aurata romanica, Sander lucioperca, Silurus glanis, Syngnathus abaster and Vimba vimba with only one observed individual per species 


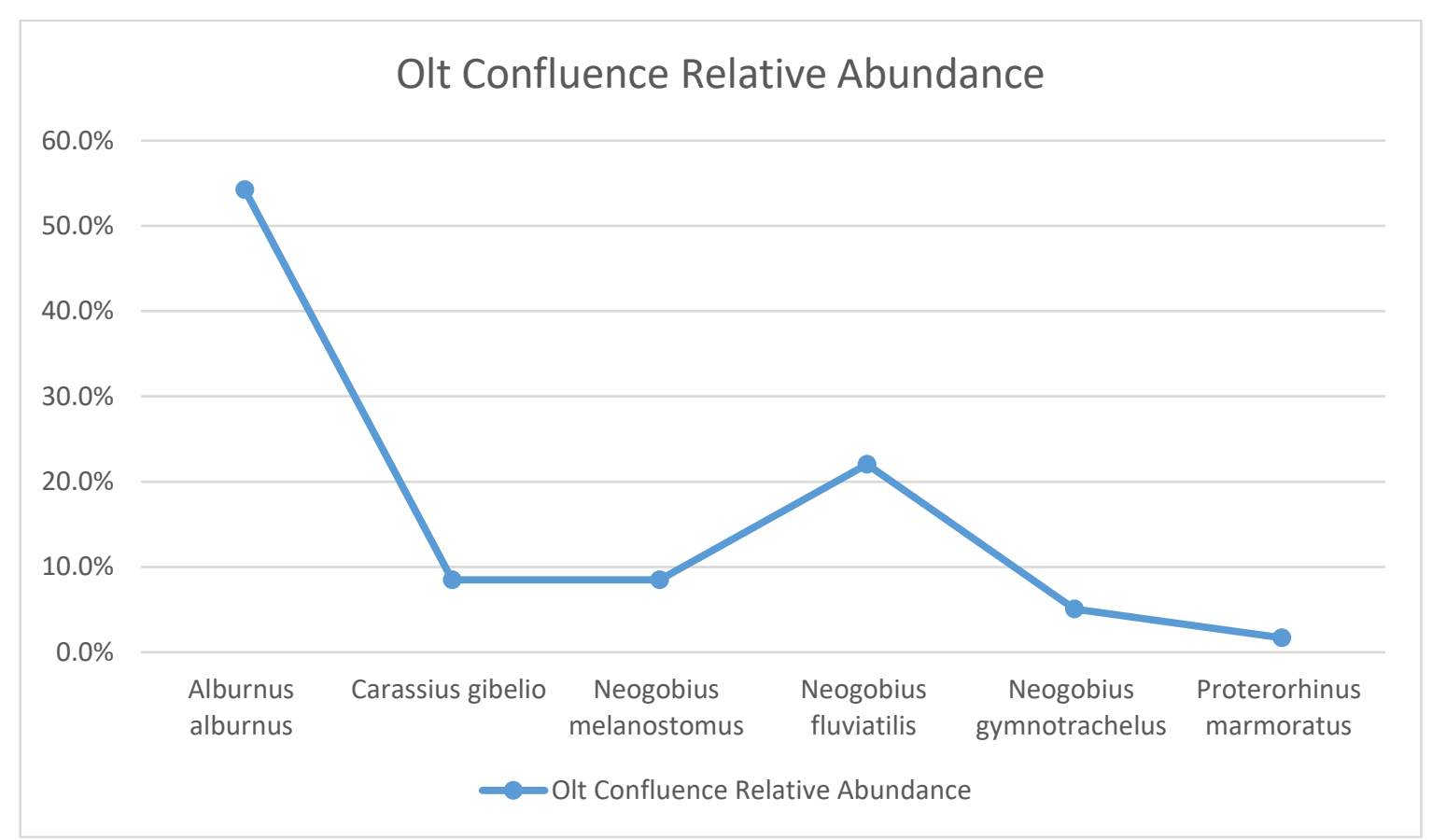

Figure 13. Relative abundance of identified fish species in shore water.

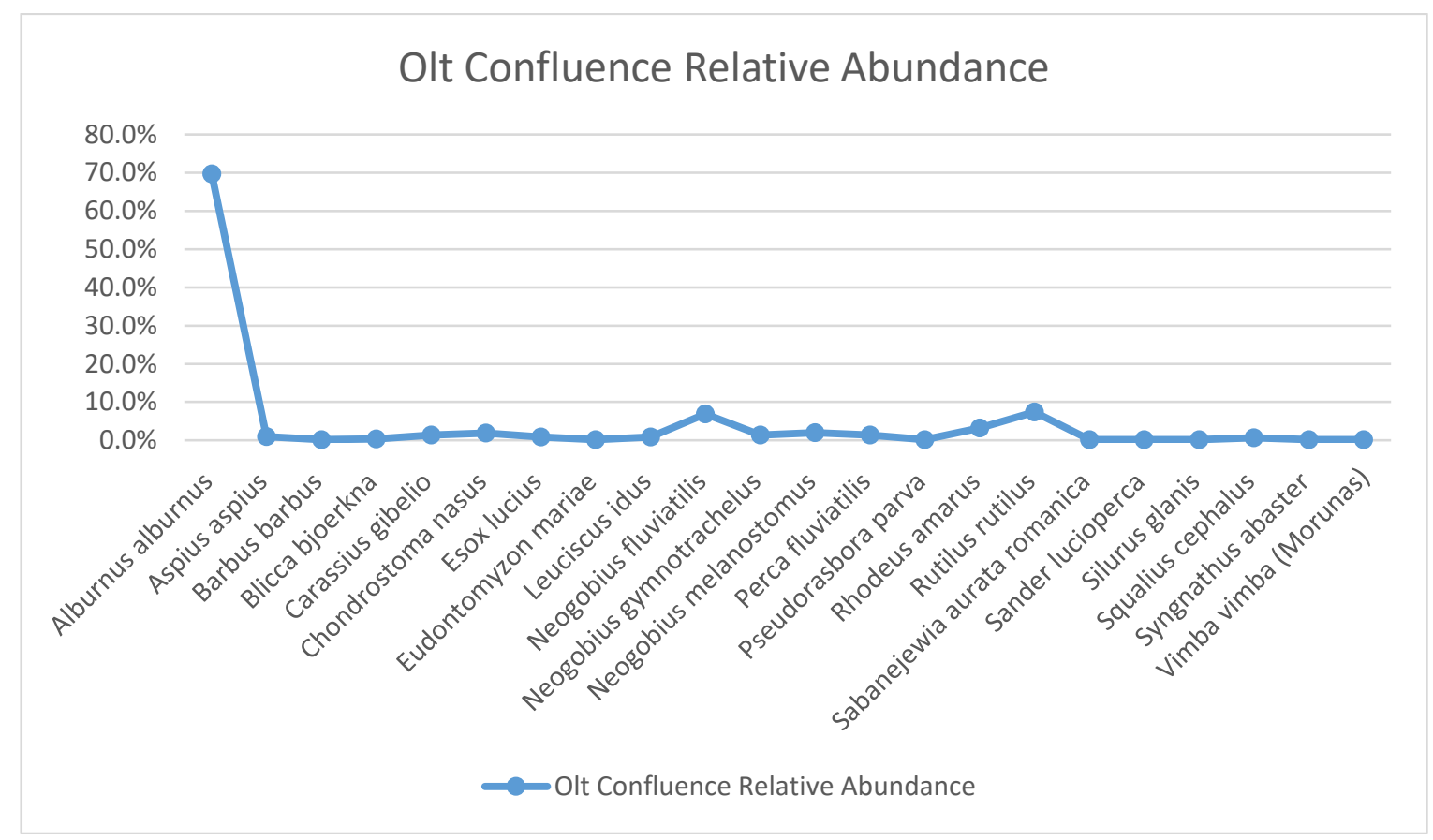

Figure 14. Relative abundance of identified fish species in open water.

\subsubsection{Belene Station}

As one of the most polluted location from a microbiological point of view, at Belene station 4 species were identified on shore water (Figure 15) and 20 species in open water (Figure 16), with 4 species of community interest: Cobitis taenia, Misgurnus fossilis, Aspius aspius and Sabanejewia aurata romanica. The dominant fish species at Belene Station were: Alburnus alburnus, Neogobius fluviatilis and Rutilus rutilus. The less frequent species for this station with only one observed specimen per species were Misgurnus fossilis. Sander lucioperca, Blicca bjoerkna, Lepomis gibbosus 
Pseudorasbora parva. For Silurus glanis 2 specimens were observed and 3 were identified as Cobitis taenia.

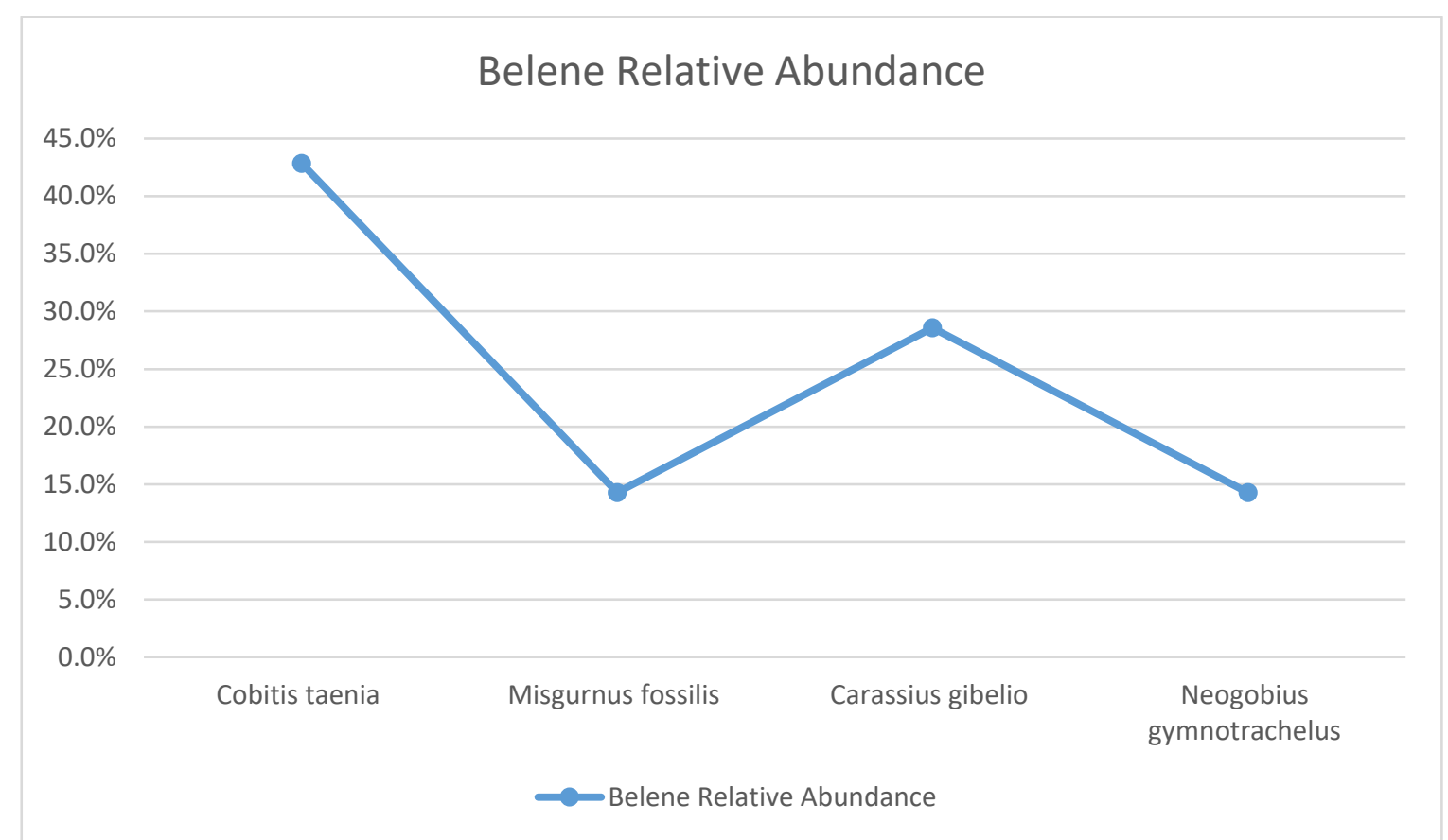

Figure 15. Relative abundance of identified fish species in shore water.

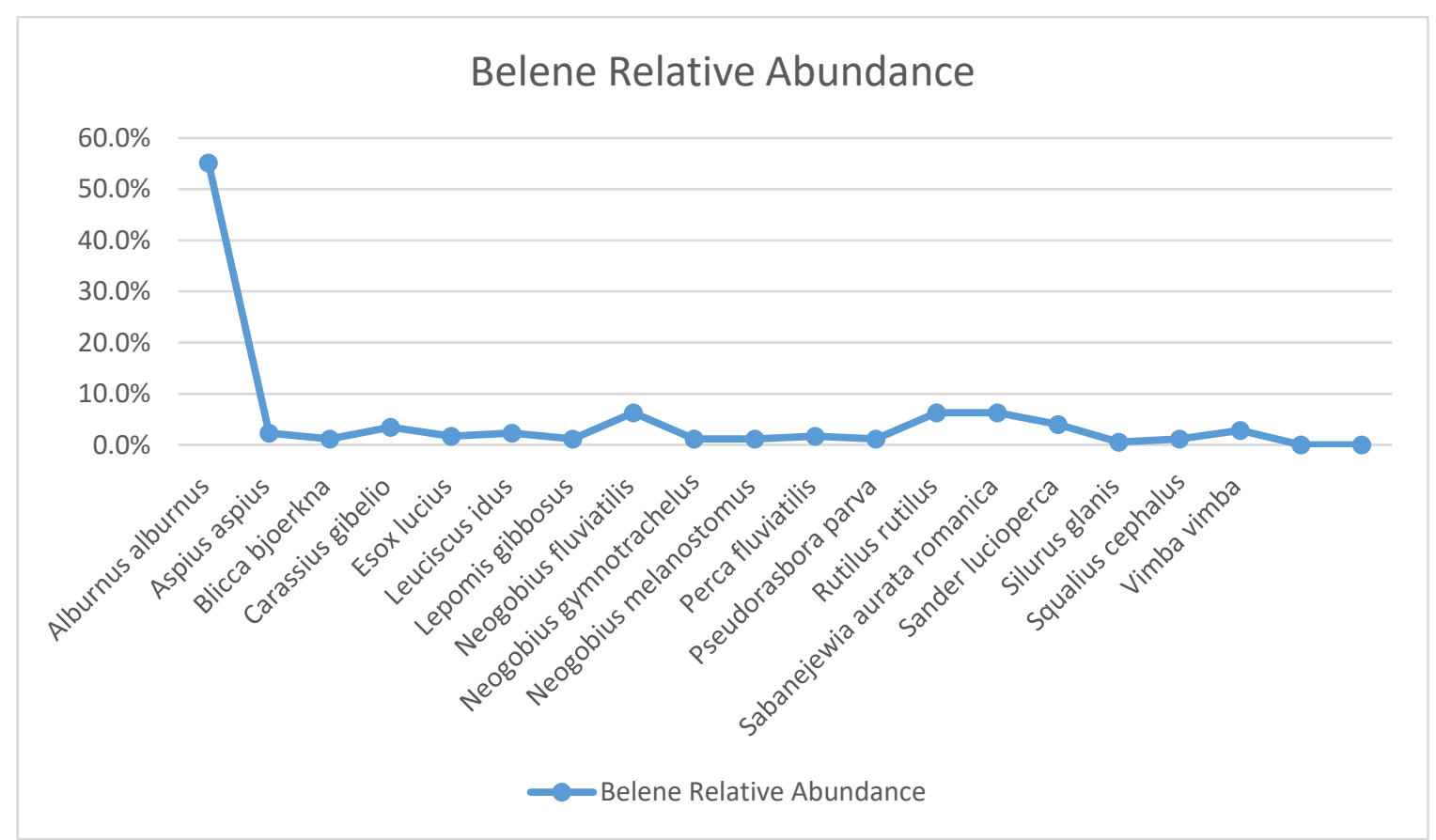

Figure 16. Relative abundance of identified fish species in open water.

\subsubsection{Vardim Station}

A total of 5 species were identified on shore water (Figure 17) and 21 species in open water (Figure 18), with 2 species of community interest: Aspius aspius and Sabanejewia aurata romanica. The dominant fish species at Vardim Station were: Alburnus alburnus followed by Sabanejewia aurata romanica and Rutilus rutilus. Less frequent species Ponticola kessleri, Pseudorasbora parva, Silurus glanis and Vimba vimba, with only one observed individual per species, were also identified. 


\section{Vardim Relative Abundance}

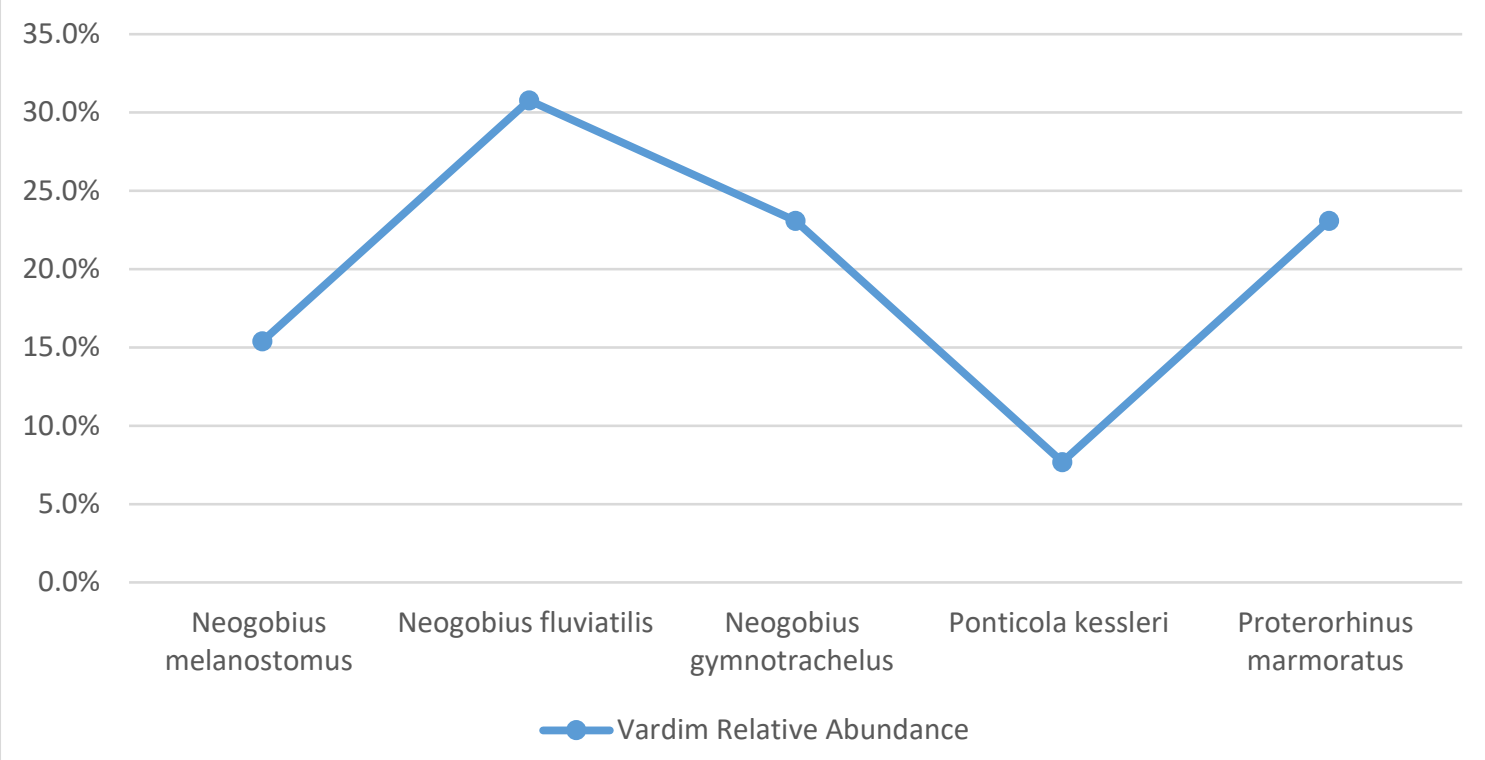

Figure 17. Relative abundance of identified fish species in shore water.

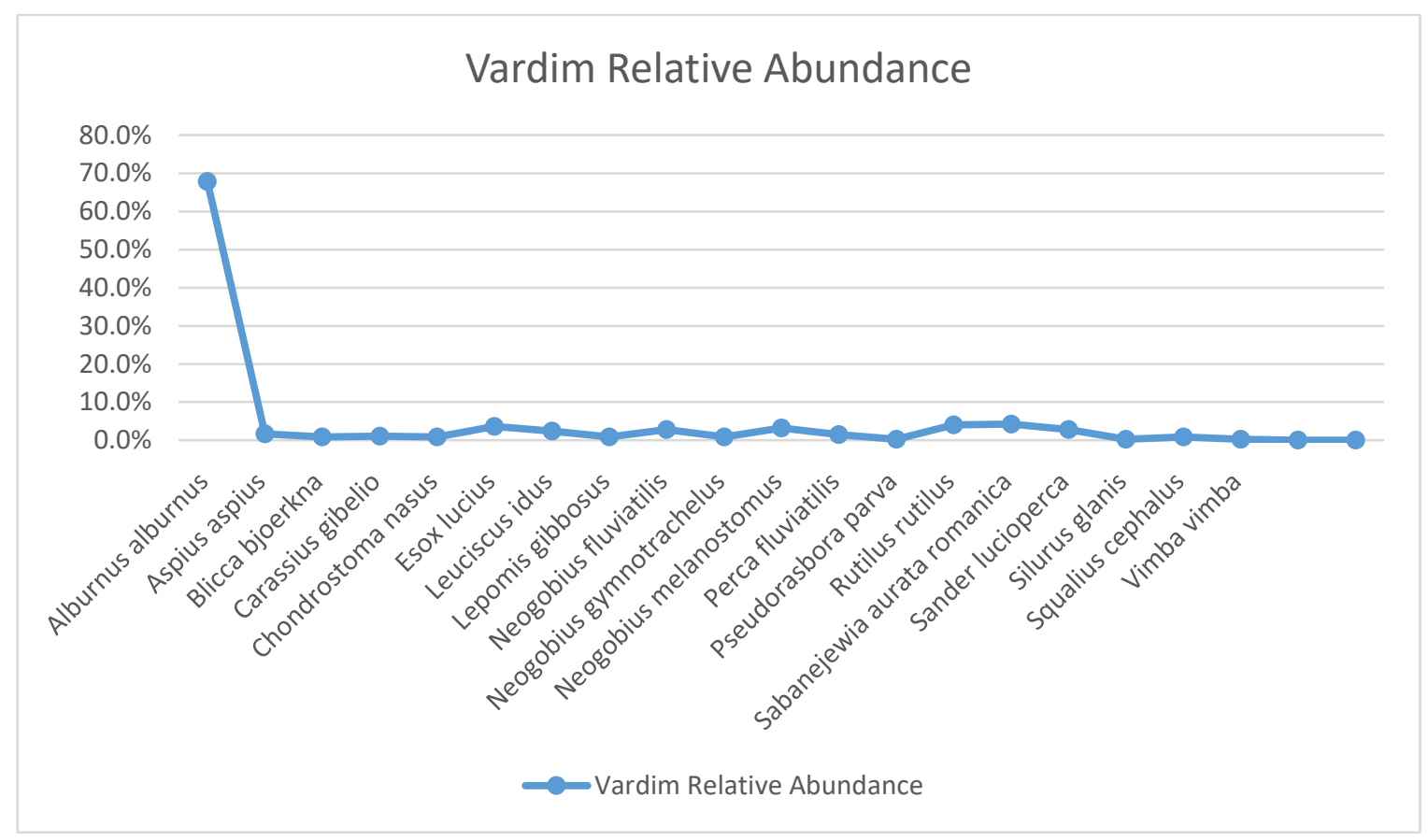

Figure 18. Relative abundance of identified fish species in open water.

\subsubsection{Iantra station}

The total number of fish species observed at this station was 6 species on shore water (Figure 19) and 14 species in open water (Figure 20), with 3 species of community interest: Aspius aspius, Rhodeus amarus and Sabanejewia aurata romanica. The dominant fish species at Iantra Station were: Alburnus alburnus, Neogobius melanostomus and Rhodeus amarus. Less frequent species found were: Esox lucius and Rutilus rutilus with only one observed specimen per species. 


\section{lantra Relative Abundance}

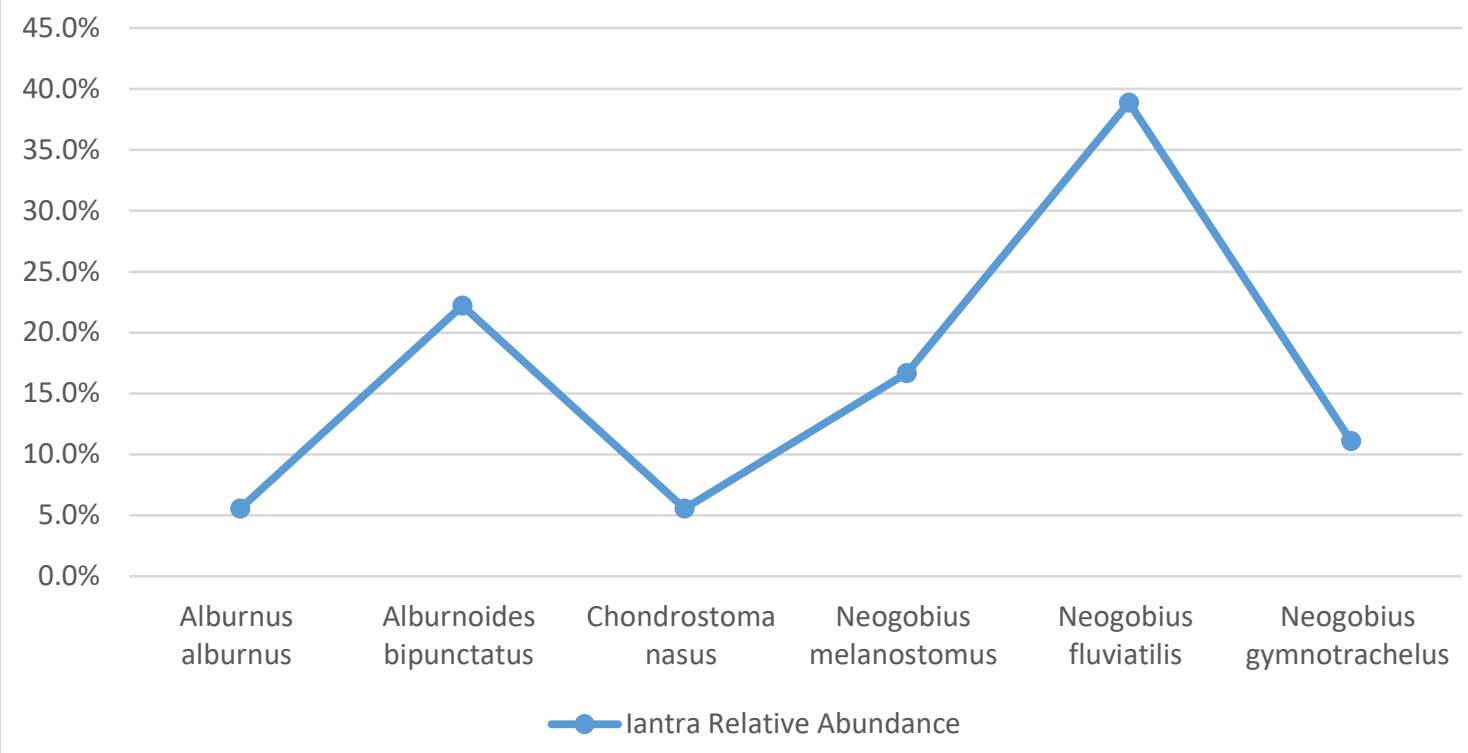

Figure 19. Relative abundance of identified fish species in shore water.

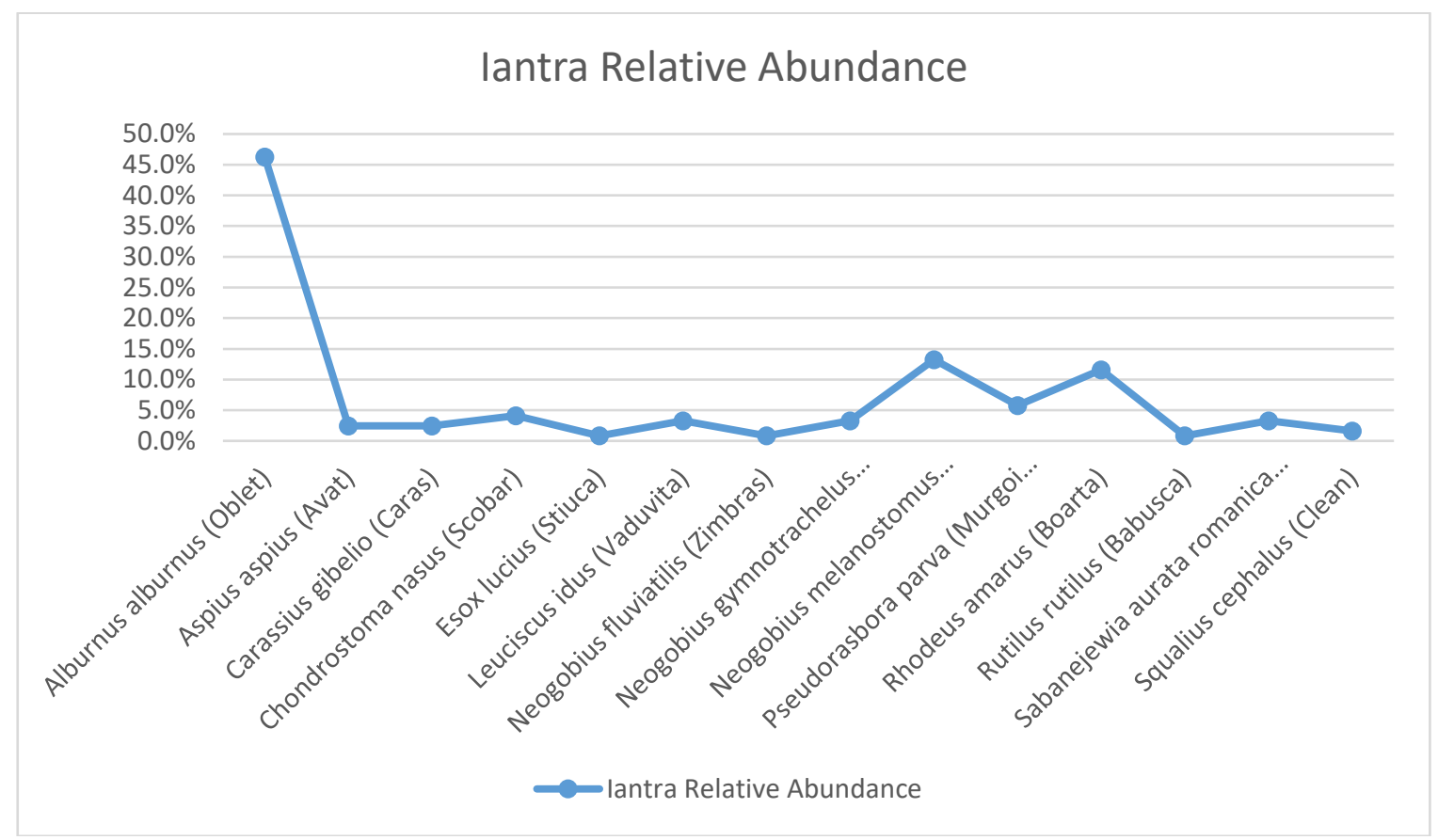

Figure 20. Relative abundance of identified fish species in open water.

\subsubsection{Batin Station}

Batin station capture on shore water was of only 4 specimens belonging to 4 different species (Figure 21) and 18 species in open water (Figure 22). Species of community interest found were Aspius aspius, Rhodeus amarus, Eudontomyzon mariae and Barbus barbus. The dominant fish species was: Alburnus alburnus, Neogobius melanostomus, Blicca bjoerkna and Rutlis rutilus. Less frequent species for this station with only one observed specimen per species was identified as Alburnoides bipunctatus, Cyprinus carpio, Leuciscus idus, and Rhodeus amarus. 


\section{Batin Relative Abundance}

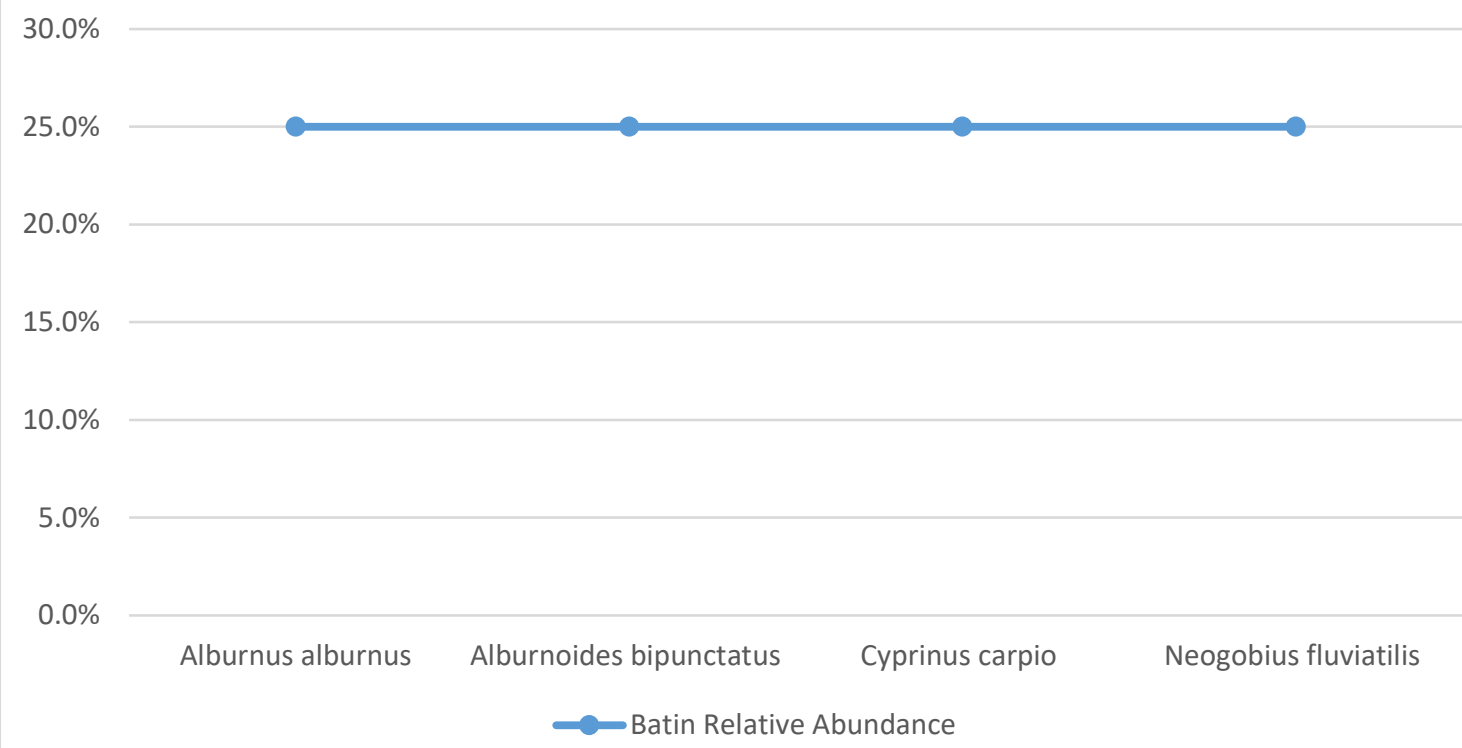

Figure 21. Relative abundance of identified fish species in shore water (only 1 specimen per species was captured).

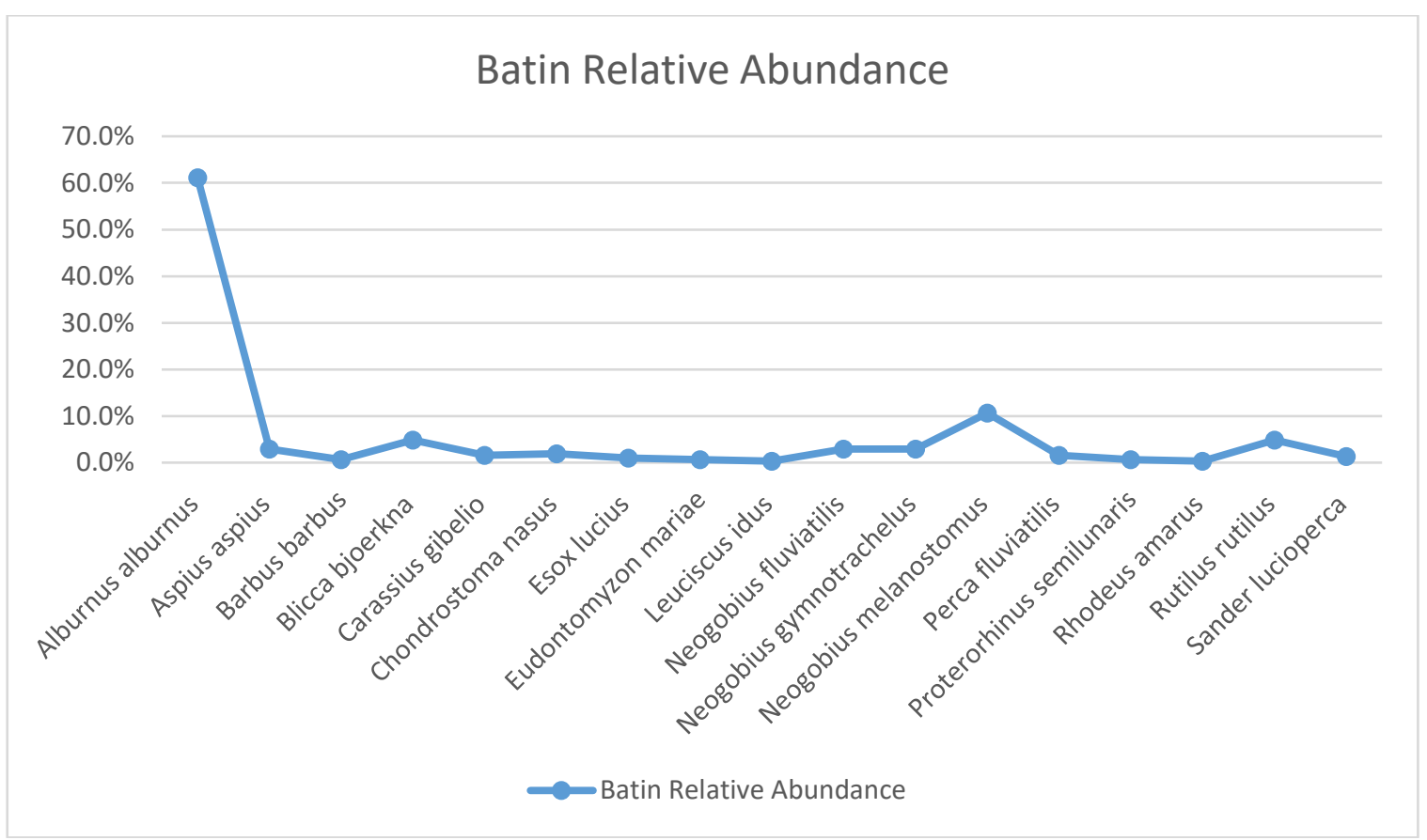

Figure 22. Relative abundance of identified fish species in open water.

\subsubsection{Kosui station}

Highly contaminated in heterotrophs and SRB, Kosui station presented a total number of 16 fish species on shore water (Figure 23) and 18 species in open water (Figure 24) study. Species of community interest found were Aspius aspius, Rhodeus amarus, Eudontomyzon mariae and Barbus barbus. The dominant fish species at Kosui Station was Neogobius melanostomus, Rhodeus amarus and Alburnus alburnus. Less frequent species for this location with only one observed specimen per species were 
Sander lucioperca, Hypophthalmichthys nobilis, Proterorhinus marmoratus, and Pseudorasbora parva.

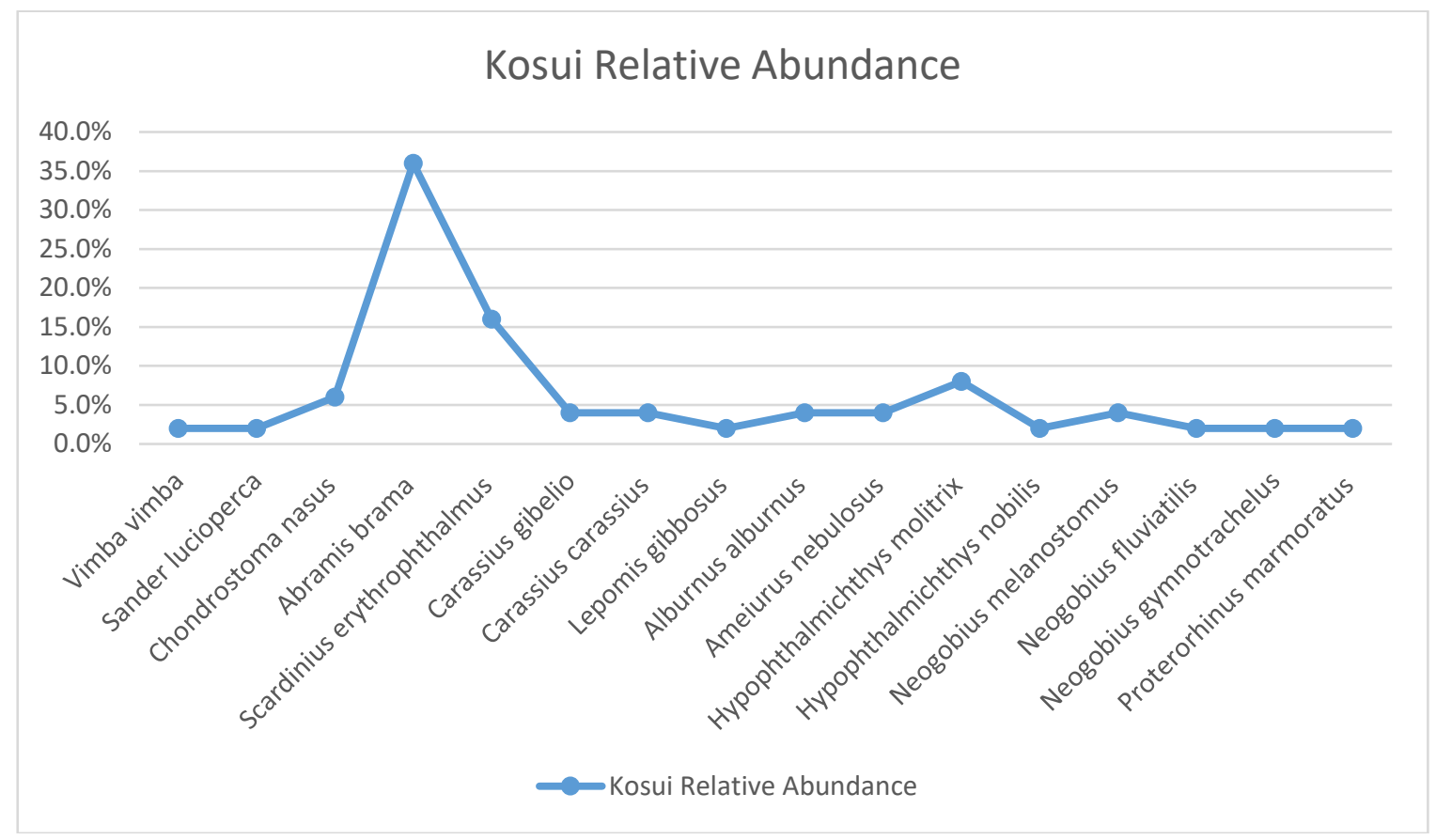

Figure 23. Relative abundance of identified fish species in shore water.

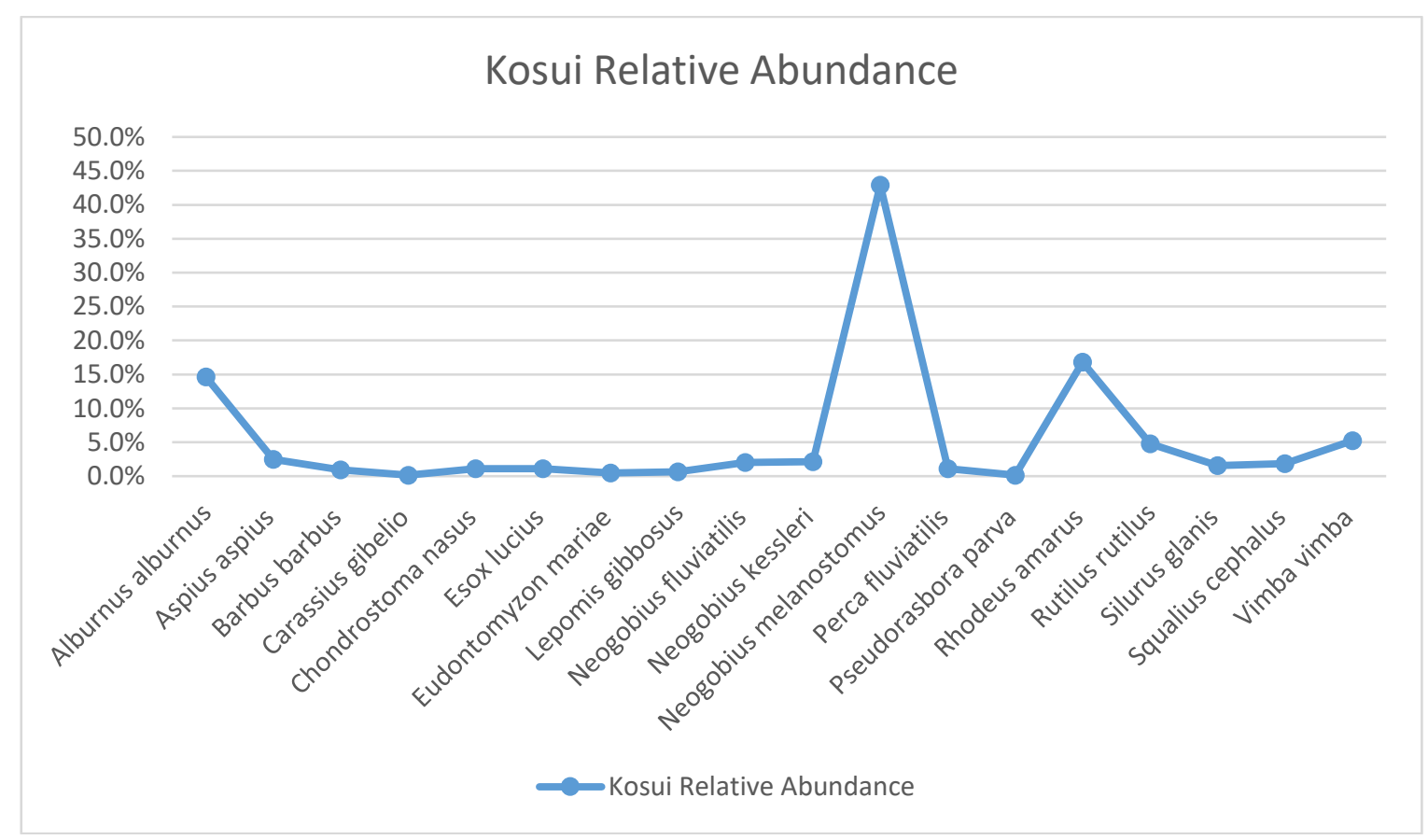

Figure 24. Relative abundance of identified fish species in open water.

\subsection{GIS Water Distribution}

The water flow oscillates depending on the factors generated by the river tributaries (Figure 2), on the precipitations in the hydrographic basin area, and also depending on the relief (Figure 3 ). The relief can determine the amount of water distributed to the tributaries or the amount of water retained in the soil. This implicitly determines the amount of nutrients and toxic elements that are in the water, as previous studies with GIS analysis regarding spatial distribution of contaminants such 
as heavy metals in the Danube River revealed (34). The flow in the Garla Mare section is decreasing up to the value of $3400 \mathrm{~m}^{3} / \mathrm{s}$, being below the multiannual average of December $\left(5200 \mathrm{~m}^{3} / \mathrm{s}\right)$.

Downstream of this sector, flows will be declining, except for the first part of the period when they will increase in the Kosui sector.

For most sediments, the particles are easily eroded by aquatic currents (35). Shields' diagram (36) allowing the delimitation of laminar transport from turbulent transport, Shields number being used to calculate the initiation of the motion of a sedimentary particle in a fluid.

The lowest values of the Shields parameter are found in the area of sands (between 0.06-2.00 $\mathrm{mm}$ ), the sand being small enough in size and mass but at the same time too large to adhere to the bed of the riverbed. Knowing that sediments also retain biotic and abiotic particles, the volume of water, the flow rate and the mass of suspended particles determine the amount of toxic elements moving in the total volume of water.

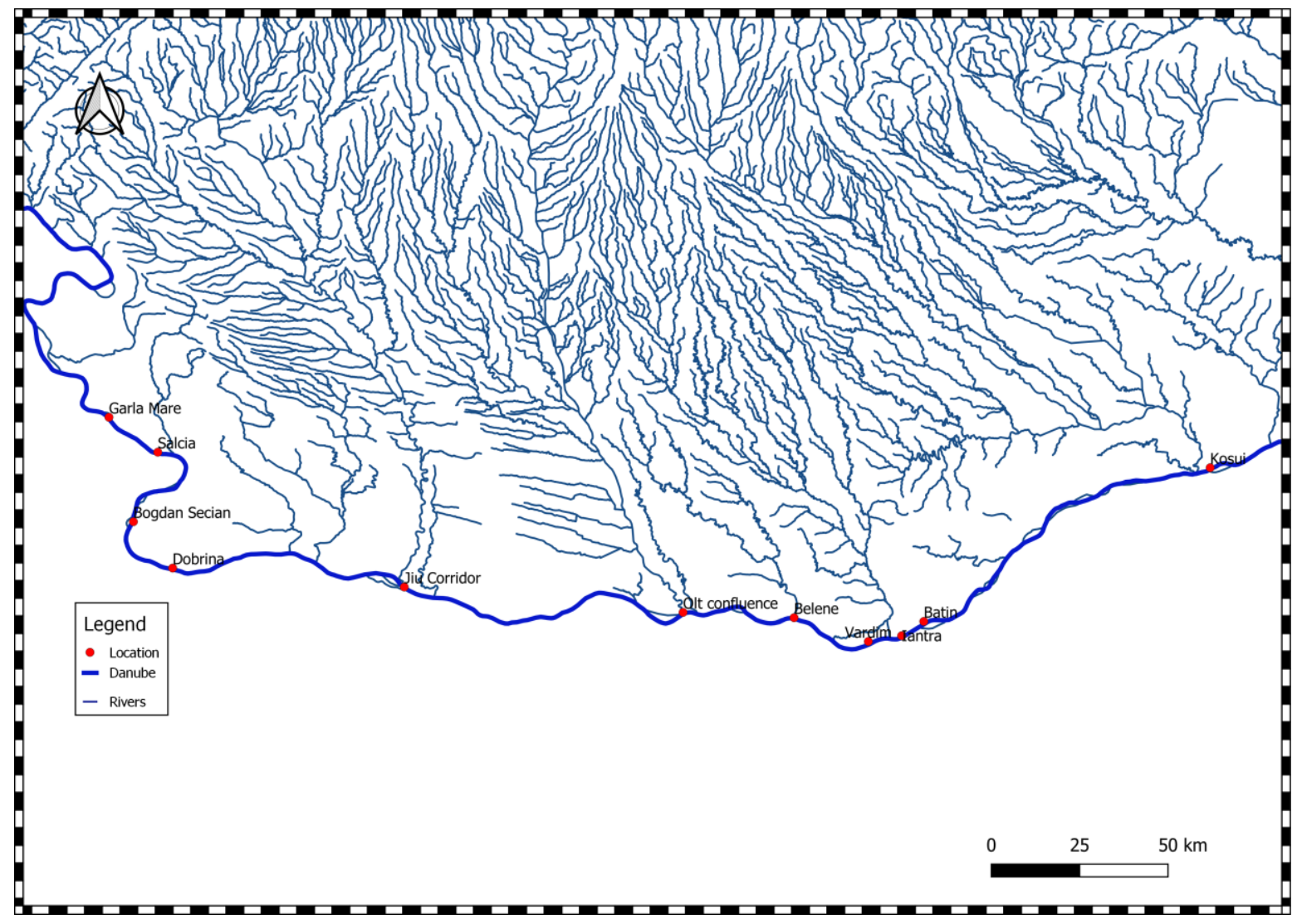

Figure 25. Hydrographic basin of the Danube Sector including tributaries. 


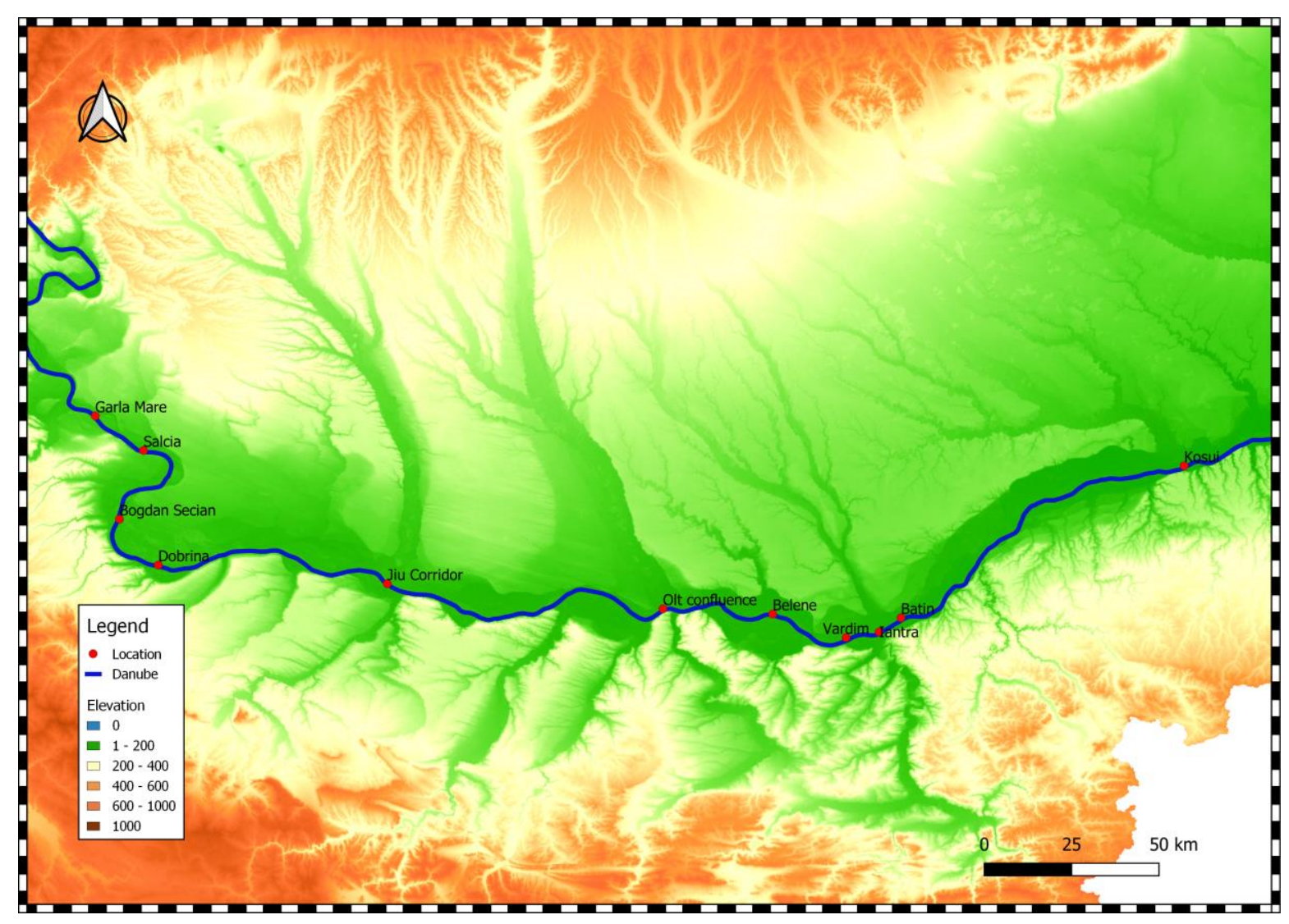

Figure 26. Hydrographic area and elevation.

\section{Conclusions}

Determining the sources responsible for both organic and inorganic pollution as well as knowing their short- and long-term effects are vital to water management and habitat safety, as well as for human health. Effective measures to counteract pollution can be taken after periodic surveys and causalities between different trophic levels as well as anthropogenic activities can be made with the use of sufficient data. Microbiology assays can provide the critical information required to identify whether the contamination is human or animal in origin as the hotspots of faecal pollution in the Danube, despite the major known contributors, can be expected to be from diffuse rather than point sources.

The sites with the highest faecal contamination indicators were Belene (S7), Kosui (S11) and Bogdan Secian (S3), with vast ichthyofauna diversity found in Kosui counting a total of 34 species and Belene with a total of 24 species, but relatively pour in Bogdan Secian with a total of 10 species.

High heterotrophic bacteria densities were found in sites with vast ichthyofauna diversity as in: Batin (S10) that shown a total of 22 species, Vardim (S8) with 25 species, and Garla Mare with 32 species.

Dobrina (S4) site with 14 identified species and Bogdan Secian (S3) site counted the fewest fish species and the lowest heterotrophic bacteria densities.

The most prominent density of SRB was found in Kosui (S11) where the highest number of species was also identified, however no correlation could be made with other sites as SRB were found in both high and low ichthyofauna diversity sites.

Although it remains certain that microbiota affects fish health at some degree, periodic surveys and further research is required to confirm the supposition that there 
could be a strong link between different microbial densities and ichthyofauna habitat preference in river water.

Acknowledgments: The authors would like to thank Hanganu Dorin for sampling and for in situ analysis.

Conflicts of Interest: The authors declare no conflict of interest.

Author Contributions: Conceptualization, M.-A.I. and C.-E.P.; methodology, C.C., N.C. and M.-A.I.; software, V.J.; formal analysis, M.-A.I.; investigation, N.C.; resources, N.C.; data curation, C.C.; writing-original draft preparation, C.-E.P.; writing-review and editing, C.-E.P; funding acquisition, N.C. All authors have read and agreed to the published version of the manuscript.

Funding: This research received no external funding

\section{References}

1. Róbert-Csaba Begy SK, Hedvig Simon and Claudiu Tănăselia. The history of the sedimentation processes and heavy metal pollution in the Central Danube Delta (Romania) in: Geochronometria Volume 45 Issue 1 (2018). 2020. doi: 10.1515/geochr-2015-0090.

2. G G, C P, F B, AE G, M S. The ecological risk of heavy metals in sediment from the Danube Delta. Ecotoxicology (London, England). 2016;25(4). doi: 10.1007/s10646-016-1627-9. PubMed PMID: 26944291.

3. Michałowicz J. Sources, toxicity and biotransformation. Environmental Toxicology and Pharmacology. Environmental Toxicology and Pharmacology; 2014. p. 738758.

4. Lamouroux N, Capra H, Souchon Y. Fish habitat preferences in large streams of southern France. Freshwater Biology. 2020;42(4):673-87.

5. Z J, N X, B L, L Z, J W, C W, et al. Metal concentrations and risk assessment in water, sediment and economic fish species with various habitat preferences and trophic guilds from Lake Caizi, Southeast China. Ecotoxicology and environmental safety. 2018;157. doi: 10.1016/j.ecoenv.2018.03.078. PubMed PMID: 29605640.

6. Jacquin L, Petitjean Q, Côte J, Laffaille P, Jean S. Effects of Pollution on Fish Behavior, Personality, and Cognition: Some Research Perspectives. Frontiers in Ecology and Evolution. 2020;8. doi: 10.3389/fevo.2020.00086.

7. Xiong JB, Nie L, Chen J. Current understanding on the roles of gut microbiota in fish disease and immunity. Zool Res. 2019;40(2):70-6. Epub 2018/07/07. doi: 10.24272/j.issn.2095-8137.2018.069. PubMed PMID: 29976843; PubMed Central PMCID: PMCPMC6378566.

8. Sylvain F, Cheaib B, Llewellyn M, Gabriel Correia T, Barros Fagundes D, Luis Val A, et al. pH drop impacts differentially skin and gut microbiota of the Amazonian fish tambaqui (Colossoma macropomum). Sci Rep. 2016;6:32032. Epub 2016/08/19. doi: 10.1038/srep32032. PubMed PMID: 27535789; PubMed Central PMCID: PMCPMC4989189.

9. Round JL, Mazmanian SK. The gut microbiota shapes intestinal immune responses during health and disease. Nat Rev Immunol. 2009;9(5):313-23. Epub 2009/04/04. doi: 10.1038/nri2515. PubMed PMID: 19343057; PubMed Central PMCID: PMCPMC4095778.

10. de Bruijn I, Department of Microbial Ecology NIoEN-K, Droevendaalsesteeg 10, Wageningen 6708PB, The Netherlands, Liu Y, Department of Microbial Ecology NIoEN-K, Droevendaalsesteeg 10, Wageningen 6708PB, The Netherlands, Wiegertjes GF, Cell Biology and Immunology group 
DoAS, Wageningen University \& Research, De Elst 1, Wageningen 6708WD, The Netherlands, et al. Exploring fish microbial communities to mitigate emerging diseases in aquaculture. FEMS Microbiology Ecology. 2020;94(1). doi: 10.1093/femsec/fix161.

11. G R, EK M, WZ S, DM P, CM C, K G, et al. Evidence for a core gut microbiota in the zebrafish. The ISME journal. 2011;5(10). doi: 10.1038/ismej.2011.38. PubMed PMID: 21472014.

12. KE S, SD E, CA L, MP OC, GL R, R K, et al. Environmental and ecological factors that shape the gut bacterial communities of fish: a meta-analysis. Molecular ecology. 2012;21(13). doi: 10.1111/j.1365-294X.2012.05552.x. PubMed PMID: 22486918.

13. S W, JF R. Intestinal microbiota composition in fishes is influenced by host ecology and environment. Molecular ecology. 2012;21(13). doi: 10.1111/j.1365-294x.2012.05646.x. PubMed PMID: 22916346.

14. Kleinhappel TK, UK SoLSUoLL, Burman OHP, UK SoLSUoLL, John EA, UK SoLSUoLL, et al. The impact of water $\mathrm{pH}$ on association preferences in fish. Ethology. 2020;125(4):195-202.

15. Jian Huang LH, Zhiqiang $\mathrm{Wu}$, Yuanmin Mo, Qi Zou, Naicheng Wu and Zhongbing Chen. Correlation of Fish Assemblages with Habitat and Environmental Variables in a Headwater Stream Section of Lijiang River, China. 2020.

16. Tesfay S, Teferi M, Tsegazeabe HH. Habitat selectivity of fresh water fishes of two secondorder tropical streams in Tigray, Northern Ethiopia. Journal of Ecology and Environment. 2019;43(1):1-11. doi: doi:10.1186/s41610-019-0107-6.

17. BLANCK A, CEMAGREF DBdEA, Lyon, France, TEDESCO PA, Institut d'Ecologia Aquàtica UdG, Girona, Spain, LAMOUROUX N, CEMAGREF DBdEA, Lyon, France. Relationships between life-history strategies of European freshwater fish species and their habitat preferences. Freshwater Biology. 2020;52(5):843-59.

18. Muniz IP. Freshwater acidification: its effects on species and communities of freshwater microbes, plants and animals I Proceedings of the Royal Society of Edinburgh, Section B: Biological Sciences | Cambridge Core. 2020. doi: doi:10.1017/S0269727000005364.

19. Jason T. Payne JJM, Colin R.Jackson, Clifford A.Ochs. Patterns of variation in diversity of the Mississippi river microbiome over 1,300 kilometers. Pone. 2017. doi: 10.1371/journal.pone.0174890.

20. Ren Z, State Key Laboratory of Simulation and Regulation of Water Cycle in River Basin CloWRaHR, Beijing, China, Flathead Lake Biological Station UoM, Polson, MT, USA, Qu X, State Key Laboratory of Simulation and Regulation of Water Cycle in River Basin CIoWRaHR, Beijing, China, Department of Water Environment CIoWRaHR, Beijing, China, et al. Functional properties of bacterial communities in water and sediment of the eutrophic river-lake system of Poyang Lake, China. PeerJ. 2019;7. doi: 10.7717/peerj.7318.

21. Cotner JB, Biddanda BA. Small Players, Large Role: Microbial Influence on Biogeochemical Processes in Pelagic Aquatic Ecosystems. Ecosystems. 2020;5(2):105-21. doi: doi:10.1007/s10021-0010059-3.

22. Van Kessel MAHJ, Mesman RJ, Arshad A, Metz JR, Spanings FAT, Van Dalen SCM, et al. Branchial nitrogen cycle symbionts can remove ammonia in fish gills. Environmental Microbiology Reports. 2016;8(5):590-4. doi: 10.1111/1758-2229.12407.

23. AR F, LF SF, SM M, FAL P. From catchment to fish: Impact of anthropogenic pressures on gill histopathology. The Science of the Total Environment. 2020;550:972-86. doi: 10.1016/j.scitotenv.2016.01.199. PubMed PMID: 26851883. 
24. WO G, OW P. Drug resistance of coliform bacteria in hospital and city sewage. Antimicrobial agents and chemotherapy. 1973;3(2). doi: 10.1128/aac.3.2.175. PubMed PMID: 4597713.

25. Sanyal S, Banerjee S. Transferable drug-resistant coliforms in fish exposed to sewage. Archives of Polish Fisheries. 2013;21(1). doi: 10.2478/aopf-2013-0004.

26. Marinescu F, Marutescu L, Savin I, Lazar V. Antibiotic resistance markers among Gramnegative isolates from wastewater and receiving rivers in South Romania. Romanian Biotechnological Letters. 2015;20:10055-69.

27. ISO 7899-2:2000(en), Water quality - Detection and enumeration of intestinal enterococci Part 2: Membrane filtration method 2020. Available from: https://www.iso.org/obp/ui/\#iso:std:iso:7899:-2:ed-2:v1:en.

28. Makori AJ, Abuom PO, Kapiyo R, Anyona DN, Dida GO. Effects of water physico-chemical parameters on tilapia (Oreochromis niloticus ) growth in earthen ponds in Teso North Sub-County, Busia County. Fisheries and Aquatic Sciences. 2017;20(1):1-10. doi: doi:10.1186/s41240-017-0075-7.

29. Nyanti L, Noor-Azhar N-I, Soo C-L, Ling T-Y, Sim S-F, Grinang J, et al. Physicochemical Parameters and Fish Assemblages in the Downstream River of a Tropical Hydroelectric Dam Subjected to Diurnal Changes in Flow. International Journal of Ecology. 2018;2018. doi: https://doi.org/10.1155/2018/8690948.

30. Gerhard G. Kavka GDK, and Andreas H. Farnleitner. Microbiological water quality of the River Danube ( $\mathrm{km} 2581$ - km 15): Longitudinal variation of pollutionas determined by standard parameters. Austrian Committee Danube Research/IAD (2006); Vienna, Austria2006. p. pp. 415-21.

31. P P, E C, G P. Blood agar to detect virulence factors in tap water heterotrophic bacteria. Applied and Environmental Microbiology. 2020;60(4):1179-83. doi: 10.1128/aem.60.4.1179-1183.1994. PubMed PMID: 8017913.

32. Marangoni PRD, Robl D, Berton MAC, Carlos Mario, Bozza A, Porsani MV, Dalzoto PdR, et al. Occurrence of sulphate reducing bacteria (SRB) associated with biocorrosion on metallic surfaces in a hydroelectric power station in Ibirama (SC) - Brazil. Braz arch biol technol. 2013;56(5):801-9. doi: 10.1590/S1516-89132013000500011.

33. HA V, LK H. Microbiologically influenced corrosion: looking to the future. International microbiology : the official journal of the Spanish Society for Microbiology. 2005;8(3). PubMed PMID: 16200495.

34. Ilie M, Marinescu F, Ghita G, Anghel A-M, Tociu C, Popescu I, et al. Spatial Distribution of Heavy Metal Contamination and Ecological Risk Assessment in Water from the Danube River. International Journal of Education and Learning Systems. 2017;02.

35. Inman DL. Sorting of sediments in the light of fluid mechanics. Journal of Sedimentary Research. 2020;19(2):51-70. doi: 10.1306/D426934B-2B26-11D7-8648000102C1865D.

36. Shields A. Anwendung der Aehnlichkeitsmechanik und der Turbulenzforschung auf die Geschiebebewegung. PhD Thesis Technical University Berlin. 2020. 


\section{Authors:}

Mădălina-Andreea Ivan, Carmen Curuțiu; Department of Microbiology, Faculty of Biology, University of Bucharest, 1-3 Aleea Portocalelor Str., 60101 Bucharest, Romania; m.ivan20@s.bio.unibuc.ro (M.-A.I.); carmen.curutiu@bio.unibuc.ro (C.C.);

Nicolae Craciun; Zoology Section, Department of Biochemistry and Molecular Biology, Faculty of Biology, University of Bucharest, 91-95 Splaiul Independenței Str., 050095, Bucharest, Romania; nicolae.craciun@yahoo.com

Valentin Jujea; Department of Geographic Information Systems, Faculty of Geography, University of Bucharest, 1 Nicolae Bălcescu Str., 010041, Bucharest, Romania; valentin.jujea@s.unibuc.ro

Cristian-Emilian Pop; Department of Organic Chemistry, Biochemistry and Catalysis, Faculty of Chemistry, University of Bucharest, 90 Panduri Str., 050663, Bucharest, Romania; cristian.pop@s.unibuc.ro 